\title{
A Low-Prevalence Single-Nucleotide Polymorphism in the Sensor Kinase PhoR in Mycobacterium tuberculosis Suppresses Its Autophosphatase Activity and Reduces Pathogenic Fitness: Implications in Evolutionary Selection
}

OPEN ACCESS

Edited by:

George Grant

University of Aberdeen,

United Kingdom

Reviewed by:

Amanda Jane Gibson, Aberystwyth University,

United Kingdom

Roberto Zenteno-Cuevas,

Universidad Veracruzana, Mexico

${ }^{*}$ Correspondence:

Deepak Kumar Saini

deepaksaini@iisc.ac.in

Specialty section:

This article was submitted to

Infectious Diseases,

a section of the journal

Frontiers in Microbiology

Received: 13 June 2021

Accepted: 30 July 2021

Published: 25 August 2021

Citation:

Waturuocha UW, Krishna MS Malhotra V, Dixit NM and Saini DK (2021) A Low-Prevalence Single-Nucleotide Polymorphism in

the Sensor Kinase PhoR

in Mycobacterium tuberculosis

Suppresses Its Autophosphatase Activity and Reduces Pathogenic Fitness: Implications in Evolutionary

Selection.

Front. Microbiol. 12:724482. doi: 10.3389/fmicb.2021.724482

\section{Uchenna Watson Waturuocha ${ }^{1,2}$, M. S. Krishna ${ }^{1}$, Vandana Malhotra ${ }^{3}$, Narendra M. Dixit ${ }^{4,5}$ and Deepak Kumar Saini ${ }^{2,5 *}$}

\footnotetext{
${ }^{1}$ Department of Studies in Zoology, University of Mysore, Mysore, India, ${ }^{2}$ Department of Molecular Reproduction Development and Genetics, Indian Institute of Science, Bengaluru, India, ${ }^{3}$ Sri Venkateswara College, University of Delhi, New Delhi, India, ${ }^{4}$ Department of Chemical Engineering, Indian Institute of Science, Bengaluru, India, ${ }^{5}$ Center for Biosystems Science and Engineering, Indian Institute of Science, Bengaluru, India
}

The genome sequencing of Mycobacterium tuberculosis, the causative organism of tuberculosis, has significantly improved our understanding of the mechanisms that drive the establishment of infection and disease progression. Several clinical strains of M. tuberculosis exhibit single-nucleotide polymorphisms (SNPs), the implications of which are only beginning to be understood. Here, we examined the impact of a specific polymorphism in PhoR, the sensor kinase of the PhoPR two-component system. Biochemical analysis revealed reduced autophosphatase/ATPase activity, which led to enhanced downstream gene expression. We complemented M. tuberculosis H37Ra with the wild-type and mutant phoPR genes and characterized the strains in a cell line infection model. We provide an explanation for the low prevalence of the SNP in clinical strains ( $1 \%)$, as the mutation causes a survival disadvantage in the host cells. The study provides a rare example of selection of a signaling node under competing evolutionary forces, wherein a biochemically superior mutation aids bacterial adaptation within-host but has low fitness for infection and hence is not selected. Our study highlights the importance of accounting for such SNPs to test therapeutic and co-therapeutic methods to combat TB.

Keywords: single-nucleotide polymorphism, two-component system, histidine kinase, Mycobacterium tuberculosis, evolutionary fitness, bacterial signal transduction, PhoR

\section{INTRODUCTION}

Sequencing of Mycobacterium tuberculosis $\mathrm{H} 37 \mathrm{Rv}$ genome, a virulent laboratory strain, has revolutionized the research done on this pathogen world over (Cole et al., 1998). It revealed the presence of sizeable unknown gene sets, like serine-threonine protein kinases (STPKs) and other relatively small groups of typical bacterial signaling systems, the two-component systems 
(TCSs). Over the years, a large number of virulent clinical strains, non-human pathogenic strains, and non-pathogenic mycobacterial strains have been sequenced, aimed at unraveling what makes $M$. tuberculosis such a successful pathogen (Cole et al., 1998; Garnier et al., 2003; Zheng et al., 2008; Ioerger et al., 2010; Tekwu et al., 2014; Mohan et al., 2015; Manson et al., 2017; Borrell et al., 2019). The findings have revealed many differences; however, their contribution toward pathogenicity is not clear (Ioerger et al., 2010).

Given the pathogenic nature of M. tuberculosis, it is expected to face adverse environments within its host (Chai et al., 2018). To adapt and survive in changing environmental conditions, it uses signaling systems called TCSs, which regulate the expression of many genes in response to various environmental stimuli (Pang et al., 2007). TCSs are typically composed of a sensor kinase (SK) as the first component that senses the external stimulus and undergoes activation by an autophosphorylation event at a specific histidine (His) residue. The second component of a TCS is a response regulator (RR), which the SK activates by a unique phosphotransfer event on a conserved aspartate (Asp) residue (Stock et al., 2000; Bourret and Silversmith, 2010). The RRs are DNA-binding transcription factors that activate or repress the transcription of downstream genes and bring about adaptive changes in the bacterium. Among the 12 pairs of TCSs in M. tuberculosis, the PhoPR TCS is extensively studied and implicated in regulating various processes, such as virulence and growth upon infection (Pérez et al., 2001; Walters et al., 2006; Ryndak et al., 2014), lipid biosynthesis (Walters et al., 2006), hypoxia (Vashist et al., 2018), pH sensing, and adaptation (Johnson et al., 2015). It is known to regulate the expression of about $2 \%$ of the M. tuberculosis genome (Cimino et al., 2012). It is essential for survival and multiplication within macrophages (Ludwiczak et al., 2002), and thus, this operon has been considered to be a master regulator in $M$. tuberculosis (Ryndak et al., 2008). We analyzed the sequences of several clinical strains and sought single-nucleotide polymorphisms (SNPs) primarily in TCS genes to understand the evolutionary pressures subjected to TCSs.

Comparative sequence analysis of strains from various lineages of $M$. tuberculosis sensu stricto (L1-L4 and L7) and Mycobacterium africanum (L5 and L6), which belong to the human-adapted $M$. tuberculosis complex, that diverged from strains that infect animals and now cause the disease in humans (Comas et al., 2014), and Mycobacterium bovis, revealed close to $\sim 2,000$ SNPs. These polymorphisms could be responsible for the physiological differences between them. Genomic differences among clinical strains have been associated with variations in protein and metabolite levels, post-translational modifications (Liu et al., 2014), drug susceptibility (Rouse et al., 1995), transcriptome (Homolka et al., 2010; Rose et al., 2013), and cell wall structure (Coscolla and Gagneux, 2014), along with virulence and infectivity. It is also observed that strains that have evolved more recently are more infectious, grow faster, and have lesser latency than their parental strains (Borrell et al., 2019).

A recent study showed that variations because of the passaging of laboratory strains used as reference pathogenic strains had impeded our understanding of the mycobacterial pathology
(Ioerger et al., 2010). This includes the adaptation of $\mathrm{H} 37 \mathrm{Rv}$ to laboratory conditions while remaining virulent in mice (Ioerger et al., 2010). It is believed that there are hot spots for insertions/duplications, deletions, or substitutions across the M. tuberculosis genome. Variants have also been observed in highly conserved TCS regions, such as a SNP in the DNA-binding domain of the phoP RR that attenuates the H37Ra strain (Wang et al., 2007; Chesne-Seck et al., 2008; Lee et al., 2008) and an SNP in the SK phoR, which shows functional and phenotypic changes in the clinical strain CDC1551 when compared with H37Rv (Schreuder et al., 2015), and yet another SNP in the phoR region of the $M$. bovis strain, which leads to pleiotropic changes in the production and secretion of lipids and affects virulence (Gonzalo-Asensio et al., 2014).

In the present study, we examined the role of one such polymorphism in the PhoR SK and its effect on mycobacterial signaling, virulence, and pathogenicity. Our findings demonstrate that the mutation enhanced the catalytic activity of the SK in both in vitro and in vivo conditions. The presence of the SNP in three of the 242 clinical strains examined suggests a poor evolutionary selection of this variant. We hypothesize that the mutant TCS is primed early and activates higher gene expression upon sensing of stimuli, leading to impaired survival of the mutation carrying bacilli during infection. This could explain the poor selection of the SNP in the wild. Overall, this study presents an example of the evolutionary benefits of temporally tuned signaling activity and suggests that similar competing forces may be at play with other TCSs.

\section{MATERIALS AND METHODS}

Chemicals, media, biochemicals, and protein reagents were obtained from Merck (Kenilworth, NJ, United States); protein marker was from Abcam (Cambridge, United Kingdom). Restriction enzymes were from Thermo Fisher Scientific (Waltham, MA, United States). Cloning and qRT primers were synthesized by Bioserve (Hyderabad, India); radioactive $\gamma^{32} \mathrm{P}$ ATP $(>4,000 \mathrm{Ci} / \mathrm{mmol})$ was from BRIT-Jonaki (Hyderabad, India); fetal bovine serum (FBS) from Thermo Fisher Scientific (United States); TRIzol from TaKaRa (Seoul, South Korea); 0.1$\mathrm{mm}$ zirconia beads from BioSpec Products Inc., (Bartlesville, OK, United States); iScript cDNA synthesis kit from Bio-Rad Laboratories (Hercules, CA, United States); DyNAmo Color Flash SYBR Green qPCR Kit from Thermo Fisher Scientific (United States); THP-1 monocyte cell line and H460 epithelial cell line from ATCC (Manassas, VA, United States); and H37Ra strain of M. tuberculosis from lab collection.

\section{In silico Sequence Analysis}

The sequences of 19 clinical isolates from India, reported as part of a previous study (Ramaiah et al., 2019), along with 223 sequences from BioProject PRJNA235851 (Manson et al., 2017), were analyzed and aligned using T-COFFEE and CLUSTALW (Madeira et al., 2019) using the phoR gene sequence from $\mathrm{H} 37 \mathrm{Rv}$ strain as a template. A variation from $\mathbf{g c c}$ to $\underline{\operatorname{acc}}$ at the 1,198 nucleotide of $p h o R$ gene was identified and named 
PhoR' (A400T). The global frequency of the SNP was tested in the GMTV database (Genome-Wide M. tuberculosis Variation database $)^{1}$ (Chernyaeva et al., 2014).

\section{Phylogenetic Tree Construction}

The sequence alignment of PhoR gene was used to generate a radial phylogenetic tree using the maximum-likelihood method based on the Tamura-Nei model by MEGA. Bootstrap analysis was performed with 500 replicates, with all sites being informative and without gaps (38).

\section{Recombinant Plasmid Construction and Generation of Bacterial Strains}

Cloning and overexpression of proteins were carried out in Escherichia coli strains DH10 $\beta$ and BL21 Arctic Express ${ }^{\mathrm{TM}}$ (Agilent Technologies, Santa Clara, CA, United States) grown in LB medium with $100 \mu \mathrm{g} / \mathrm{ml}$ of ampicillin or $50 \mu \mathrm{g} / \mathrm{ml}$ of gentamycin, respectively. Primers used for PCR and cloning are listed in Supplementary Table 1. Recombinant plasmids used for protein overexpression are reported previously (Agrawal et al., 2015). For generating mycobacterial expression plasmid containing the PhoPR operon with its native promoter, the nucleotide region of the phoPR operon, along with a 500-bp upstream region, was PCR-amplified from H37Rv genomic DNA using specific primers. The amplicon was cloned in pCV125 vector, a mycobacterial single copy, and integrative expression vector with kanamycin resistance marker, at NdeI and HindIII restriction sites. The recombinant constructs generated were verified by DNA sequencing.

\section{Expression and Purification of Recombinant Proteins}

The recombinant proteins were expressed and purified, as reported previously (Saini et al., 2002). In brief, E. coli cells containing expression plasmids for the SK and RR proteins were grown at $37^{\circ} \mathrm{C}$ in $200 \mathrm{ml}$ of Terrific broth (TB) to an $\mathrm{OD}_{600}>1.0$ followed by induction with IPTG $(0.1-1.0 \mathrm{mM})$. The culture was further grown for $15-20 \mathrm{~h}$ at $10^{\circ} \mathrm{C}-13^{\circ} \mathrm{C}$ for protein expression. Cells were harvested by centrifugation, and soluble $6 \times$ His-tagged proteins were purified using $\mathrm{Ni}^{+2}$-NTA, as described previously (34). The pellets were resuspended in native lysis buffer $(50 \mathrm{mM}$ of Tris- $\mathrm{Cl}, \mathrm{pH} 8,300 \mathrm{mM}$ of $\mathrm{NaCl}$, and $10 \%$ glycerol) with $1 \mathrm{mM}$ of phenylmethylsulfonyl fluoride (PMSF) and $1 \mathrm{mM}$ of benzamidine and sonicated on ice for 5-10 min at 25\% amplitude (pulse on $3 \mathrm{~s}$, pulse off $2 \mathrm{~s}$ ). The lysate was centrifuged at $14,000 \mathrm{~g}$ for $30 \mathrm{~min}$ at $4^{\circ} \mathrm{C}$. The supernatant containing the protein was passed through the $\mathrm{Ni}^{+2}$ NTA column for about 120 min with intermittent shaking on a pre-equilibrated with native lysis buffer at $4^{\circ} \mathrm{C}$ for $15 \mathrm{~min}$. The unbound proteins were discarded as flow-through, and the column was washed with wash buffer A (25 mM of Tris-Cl, $\mathrm{pH}$ $8.0,500 \mathrm{mM}$ of $\mathrm{NaCl}, 25 \mathrm{mM}$ of imidazole, and $10 \%$ glycerol) and wash buffer B (25 mM of Tris-Cl, pH 8.0, $500 \mathrm{mM}$ of $\mathrm{NaCl}$, $50 \mathrm{mM}$ of imidazole, and $10 \%$ glycerol). The bound protein was

${ }^{1}$ https://mtb.dobzhanskycenter.org eluted out with elution buffer (25 mM of Tris-Cl, pH 8.0, $500 \mathrm{mM}$ of $\mathrm{NaCl}, 250 \mathrm{mM}$ of imidazole, and $10 \%$ glycerol).

\section{Dialysis and Storage of Purified Proteins}

The eluted fractions of the proteins with the highest yield determined by Bradford assay were pooled together and dialyzed against dialysis buffer I ( $50 \mathrm{mM}$ of Tris-Cl, $\mathrm{pH} 8.0,50 \mathrm{mM}$ of $\mathrm{NaCl}, 1 \mathrm{mM}$ of DTT, and $10 \%$ glycerol) for $6-12 \mathrm{~h}$ and then with dialysis buffer II or storage buffer (50 mM of Tris-Cl, pH 8.0, $50 \mathrm{mM}$ of $\mathrm{NaCl}, 0.1 \mathrm{mM}$ of DTT, and $50 \%$ glycerol) overnight. The concentration of the purified proteins after dialysis was determined by Bradford assay using bovine serum albumin (BSA) as standard. The purity of the proteins was checked on sodium dodecyl sulfate-polyacrylamide gel electrophoresis (SDS-PAGE) and stored at $-20^{\circ} \mathrm{C}$.

\section{Circular Dichroism Spectroscopy}

PhoR wt and PhoR' (A400T) proteins were subjected to circular dichroism spectroscopy using JASCO J-810 Spectropolarimeter. Spectra for SK protein (in $1 \times \mathrm{PBS}$ ) were analyzed between wavelength 190 and $300 \mathrm{~nm}$ to record protein secondary structures. The K2D3 software was used to analyze the plots.

\section{Phosphorylation Assays}

Autophosphorylation assays were performed as described previously (Agrawal et al., 2015). Briefly, $50 \mathrm{pmol}$ of the purified SKs [PhoR wt and PhoR' (A400T)] were autophosphorylated in kinase buffer (50 mM of Tris-Cl (pH 8.0), $50 \mathrm{mM}$ of $\mathrm{KCl}$, and $20 \mathrm{mM}$ of $\left.\mathrm{MgCl}_{2}\right), 100 \mu \mathrm{M}$ of $\mathrm{ATP}$, and $2 \mu \mathrm{Ci}$ of $\gamma^{32} \mathrm{P}$-ATP $(>4,000 \mathrm{Ci} / \mathrm{mmol})$ for $60 \mathrm{~min}$ at $30^{\circ} \mathrm{C}$. The reaction was terminated using a $1 \times$ SDS-PAGE buffer and resolved on a $12.5 \%$ SDS-PAGE gel. The gels were washed and exposed to a phosphor screen (Fujifilm, Tokyo, Japan), followed by imaging with Typhoon phosphorimager (GE Healthcare, Chicago, IL, United States). Images were adjusted for brightness and contrast with the Microsoft image editing tool, and quantitative densitometric analysis of the autoradiograms was done using ImageJ software. The first time point's signal was considered 100\%, and relative levels of phosphorylation over time with the wild-type (WT) and mutant SKs were determined to quantify the effects of the mutation. For statistical analysis, significance ( $p$-values) were calculated regarding $\mathrm{SK} \sim \mathrm{P}$ levels in the autophosphorylation reaction. In the case of the autophosphorylation of the SK in the presence of the PhoR inhibitor, ethoxzolamide (ETZ) (Johnson et al., 2015), the WT and mutant SKs were incubated for $2 \mathrm{~h}$ with increasing concentrations of the inhibitor in kinase buffer (described above) at $30^{\circ} \mathrm{C}$. The reaction was terminated using a $1 \times$ SDS-PAGE loading buffer, loaded on an SDS-PAGE gel, and processed as described above. In the phosphotransfer assay, 150 pmol of the RR diluted in kinase buffer was added to the autophosphorylation reaction, containing $50 \mathrm{pmol}$ of phosphorylated SK (for $60 \mathrm{~min}$ ) and incubated for $60 \mathrm{~min}$, and the reaction was terminated using a $1 \times$ SDS-PAGE loading buffer. The samples were loaded on an SDS-PAGE gel and processed as described above. 


\section{ATP Hydrolysis and ${ }^{32}$ IP Release Assay by Thin-Layer Chromatography}

In the phosphotransfer reaction, 150 pmol of the RR diluted in kinase buffer was added to the autophosphorylation reaction of $10 \mu \mathrm{l}$ volume, containing $50 \mathrm{pmol}$ of phosphorylated SK, for the indicated times. The reaction was terminated using $50 \mathrm{mM}$ of EDTA. The amounts of labeled ${ }^{32} \mathrm{P}$ released and residual ATP in the reaction were determined by thin-layer chromatography (TLC) on polyethyleneimine-cellulose plates using $2 \mathrm{M}$ of $\mathrm{HCOOH}$ and $2 \mathrm{M}$ of $\mathrm{LiCl}(2: 1)$ as the mobile phase; the dried plates were exposed to a phosphor screen and scanned with Typhoon phosphorimager. A quantitative comparison for the amount of ${ }^{32} \mathrm{iP}$ released was made by normalizing the later time points in the presence of the $\mathrm{RR}$ to the ${ }^{32} \mathrm{iP}$ generated by the SK alone.

\section{Electrophoretic Mobility Shift Assay}

A 500-bp region corresponding to the aprA promoter region was PCR-amplified from M. tuberculosis H37Rv genomic DNA template using specific primers (Supplementary Table 1). The PCR products were purified and end-labeled with $\gamma^{32} \mathrm{P}$ phosphate using T4 polynucleotide kinase (Thermo Fisher Scientific, United States) as per the manufacturer's protocol. The labeled fragments were purified and used for electrophoretic mobility shift assay (EMSA), by incubating with the indicated amount of PhoP protein for $45 \mathrm{~min}$ at $25^{\circ} \mathrm{C}$ in the binding buffer $(25 \mathrm{mM}$ of Tris-Cl, pH 8.0, $20 \mathrm{mM}$ of $\mathrm{KCl}, 6 \mathrm{mM}$ of $\mathrm{MgCl}_{2}, 0.10 \mathrm{mg} / \mathrm{ml}$ of BSA, $0.5 \%$ glycerol, $1 \mathrm{mM}$ of DTT, $0.5 \mathrm{mM}$ of EDTA, and $1 \mu \mathrm{g}$ of poly dI.dC). SK proteins were autophosphorylated with $5 \mathrm{mM}$ of ATP and incubated with $\mathrm{PhoP}$ to obtain $\mathrm{PhoP} \sim \mathrm{P}$. The reaction mixtures were resolved on a $4 \%$ native polyacrylamide gel $(29.5: 0.5)$ and pre-equilibrated for $1-2 \mathrm{~h}$ at $80 \mathrm{~V}$ in $0.5 \times$ Tris-borate EDTA buffer at $4^{\circ} \mathrm{C}$. Electrophoresis was performed at $4^{\circ} \mathrm{C}$ at $80 \mathrm{~V}$ for $2-3 \mathrm{~h}$. The DNA-protein complexes were visualized by phosphorimaging, as described above.

\section{Analysis of aprA Promoter-Reporter Activity}

Cultures of $M$. tuberculosis H37Ra containing either the pCV125 empty vector or the WT or mutant $p h o P R$ construct were electroporated with the aprA':GFP, smyc':mCherry (obtained as a kind gift from Prof. R. Abramovitch, United States) promoterreporter construct (Abramovitch et al., 2011). The strains were grown in $10 \mathrm{ml}$ of $7 \mathrm{H} 9$ media buffered to $\mathrm{pH} 5.5$ for induction and kept at $\mathrm{pH} 7.4$ for uninduced conditions with an initial $\mathrm{OD}_{600}$ of 0.05 for 12 days for PhoR activation experiments. At each time point, samples from each culture were taken in a 96-well flat black clear-bottom plate (Corning Inc., New York, NY, United States). Green fluorescent protein (GFP) and mCherry fluorescence ratios were measured using the Tecan Infinite M1000 plate reader (Tecan, Grödig, Austria) in duplicates along with $\mathrm{OD}_{600}$. On the 12th day, cultures were fixed in $4 \%$ paraformaldehyde and mounted on glass slides in $10 \%$ glycerol. The relative fluorescence for $\sim 300$ bacilli from each sample was quantified by microscopy analysis from three individual experiments. In infection experiments (below), for in cellulo analysis, H460 lung epithelial cell line (ATCC) was infected at a multiplicity of infection (MOI) of 1:10; infected cells were fixed and imaged at $4 \mathrm{~h}$ post-infection using the Olympus IX83 fluorescence microscope to measure the relative GFP by mCherry ratios from the infected bacilli.

\section{RNA Extraction and Quantitative Gene Expression Analysis}

Cultures of M. tuberculosis H37Ra containing either the pCV125 empty vector backbone alone or either the WT or mutant phoPR construct were grown exponentially to an $\mathrm{OD}_{600}$ of 0.8 and harvested. For acid induction experiments, cultures were grown in $10 \mathrm{ml}$ of $7 \mathrm{H} 9$ buffered to $\mathrm{pH} 5.5$ (using $\mathrm{HCl}$ ) for induced cultures and $\mathrm{pH} 7.4$ for uninduced cultures. The pellets were resuspended in QIAzol Lysis reagent (Qiagen, Hilden, Germany), mixed with zirconia silica beads, and disrupted using a mini bead beater, following which RNA was precipitated using ethanol. The RNA obtained with $\mathrm{OD}_{260} / \mathrm{OD}_{280}$ ratios $\geq 2$ was treated with DNaseI, and 500 ng of the total RNA was reverse transcribed using random hexamers and iScript reverse transcriptase (Bio-Rad, United States) as per the manufacturer's protocol. Gene-specific qRT-PCR was performed using DyNAmo Color Flash SYBR Green qPCR Kit (Thermo Fisher Scientific, United States) in Roto-GeneQ cycler (Qiagen, Germany), using $0.5 \mu \mathrm{l}$ of the cDNA synthesized per $10-\mu \mathrm{l}$ reaction with primers from previously published studies as indicated in Supplementary Table 1. The calculated threshold cycle $(\mathrm{Ct})$ value for each gene was normalized to $16 \mathrm{~S}$ rRNA followed by that of the gene of interest in the strain containing vector-only to determine fold change. The expression analyses were performed using three independent biological replicates.

\section{Macrophage Cell Line Infections}

Infection experiments were performed as previously described (Estrella et al., 2011). Briefly, THP-1 monocytes (ATCC) differentiated into macrophages with $20 \mathrm{nM}$ of phorbol 12myristate-13-acetate were infected at an MOI of 1:10 with the three strains of H37Ra (pCV125 empty vector backbone alone or the WT or mutant phoPR construct). The bacteria were allowed to infect cells for $4 \mathrm{~h}$ (taken as the time point of invasion), after which cells were washed with PBS thrice and either lysed with Triton X-100 and plated after serial dilutions on Middlebrook 7H11-OADC plates for colony enumeration or replenished with fresh antibiotic-free Roswell Park Memorial Institute (RPMI) medium to be lysed and plated at a later time point as indicated. In the experiments with PhoR inhibitor, cells were pretreated with $80 \mu \mathrm{g}$ ETZ, which was replenished every 2 days (wherever needed), and equal volumes of DMSO was added to untreated cells, followed by lysing of the cells and plating as described above. This was repeated to obtain three biological replicates (Johnson et al., 2015).

\section{Measurement of Nitrite Production}

The nitrite produced by infected THP-1 cells was measured as described previously using the Griess reagent (37). Briefly, 
the supernatants of uninfected and infected cells were collected at indicated time points and then incubated with the Griess reagent in a 1:1 proportion at room temperature for $10 \mathrm{~min}$, and the absorbance was measured at $540 \mathrm{~nm}$ in the Tecan Infinite M1000 plate reader. The nitrite concentration was determined using a standard curve plotted with different sodium nitrite concentrations and represented as $\mu \mathrm{M} / 10^{5}$ cells per well, and normalized to the uninfected 4 -h time point of invasion and blanked with the cell-free medium. This was performed to get three biological replicates, and $p$-values (statistical significance) were calculated the Mann-Whitney test.

\section{Statistical Analyses}

Statistical analyses for significance were performed using Student's $t$-test, one-way ANOVA test, and two-way ANOVA test (for time-course experiments) between control and experimental sets and induced and uninduced sets (wherever applicable). For all experiments, the number of independent biological replicates used is indicated by " $n$."

\section{RESULTS}

Clinical isolates of $M$. tuberculosis harbor several SNPs leading to variations in their sequences compared with laboratory strains. Although the changes brought about by sequence variations in various bacterial genome regions have been characterized previously, TCS regions have seldom been studied. To check the effect of such polymorphisms on the signaling and the interactions between the bacterium and its host, we analyzed the gene sequence of the phoPR TCS.

\section{An Single-Nucleotide Polymorphism in the HATPase Domain of the Sensor Kinase PhoR Is Found in Some Clinical Strains of Mycobacterium tuberculosis}

We analyzed the sequence of $p h o R$ in 242 mycobacterial strains from BioProject PRJNA235851 in National Center for Biotechnology Information (NCBI) (Ramaiah et al., 2019) with a combination of drug-sensitive/resistant, polydrug- and multidrug-resistant cohort of a South Indian population (Ramaiah et al., 2019) with $\mathrm{H} 37 \mathrm{Rv}$ as the reference sequence to identify various SNPs, and we found one that translated into a non-synonymous, missense mutation in the protein. This SNP changed alanine to threonine residue (A400T) at the 400th position, in the HATPase domain (Figures 1A,B). The SNP was found in only three strains of the genome sequences analyzed from the cohort (Figure 1A). A schematic phylogenetic tree drawn with the PhoR sequence alignment, for the genomes analyzed, shows that the strains with the mutation A400T group separated from the rest of the strains (Supplementary Figure 1). We also analyzed the genome sequence repository database GMTV, which consists of 2,501 clinical genome sequences that have been aligned for mutations in different regions of the bacteria; however, this particular SNP was not reported there. Subsequent analyses of the frequencies of other SNPs in the
SK PhoR (Figure 1C) revealed no particular hot spots for any SNPs in the gene.

Interestingly, other TCSs in these strains have SNPs in the various regions with varied frequencies (Figure 1D). The low frequency of the mutation in our study and absence in the GMTV database made us explore the changes brought about by this mutation A400T on the catalytic activities of the SK PhoR.

\section{A400T Substitution in the Kinase Domain Alters PhoR Phosphatase Activity in vitro}

We introduced the mutation in the cytosolic C-terminal domain of the SK protein PhoR (Agrawal et al., 2015) by site-directed mutagenesis and purified both WT and mutant proteins. Predicted structure analysis of the HATPase and kinase domain of the WT and mutant protein revealed no major structural differences (Supplementary Figure 2A), and circular dichroism analysis also revealed no structural perturbations between the purified proteins (Supplementary Figure 2B), allowing us to proceed with biochemical analysis of the mutant protein. We performed autophosphorylation analysis for the WT and mutant SK proteins. The proteins were incubated with $\gamma^{32}$ P-labeled ATP, and their phosphorylation status was examined. We observed enhanced autophosphorylation for the mutant compared with the WT PhoR protein at all time points (Figures 2A,B). Next, we examined the ability of the phosphorylated WT and mutant SKs to transfer the phosphoryl moiety to the RR protein PhoP. A stable and higher phosphotransfer was observed with the mutant PhoR' (A400T) protein (Figure 2C), and on quantitation, the amount of $\mathrm{RR} \sim \mathrm{P}$ was also detected to be higher (Figure 2D).

The faint signal on the WT PhoR (Figure 2C, right) suggested an inherent phosphatase activity in the PhoR SK, which seems to be reduced in mutant $S K$ as well as in the presence of $R R$ PhoP. While the presence of a phosphatase activity in SK toward $\mathrm{RR} \sim \mathrm{P}$ is widely reported and is attributed to the DHp domain (Xing et al., 2017), the presence of an autophosphatase activity of SK is rarely reported (Dubey et al., 2016). Such an activity would result in dephosphorylation of the $\mathrm{SK} \sim \mathrm{P}$, releasing free ${ }^{32} \mathrm{iP}$. We measured the concentration of ${ }^{32} \mathrm{iP}$ species released through TLC analysis by incubating phosphorylated WT or the mutant SK in the presence or absence of the RR PhoP. The intensities were normalized to those with SK alone, and the relative ${ }^{32} \mathrm{iP}$ and ATP signal intensities of the spots at various time points were noted in the presence of the RR PhoP. Figure 2E (lanes 1 and 7) indicates two things: first, both proteins have an ATPase or autophosphatase activity, as evidenced by the amount of ${ }^{32} \mathrm{iP}$ released; and second, the mutant kinase exhibited a reduced auto-dephosphorylation ability compared with the WT protein.

We concomitantly recorded higher levels of residual ATP for the mutant PhoR' (A400T) protein (Figure 2E, right panel; lanes 7-12), compared with the WT kinase (Figure 2E, left panel; lanes 1-6). Figure $2 \mathrm{~F}$ shows a significant reduction in the amount and rate of ${ }^{32} \mathrm{iP}$ released for the mutant protein. Taken together, the data indicate that the mutant SK PhoR' (A400T) protein is catalytically more active than the WT PhoR by virtue of stable phosphotransfer and a decreased phosphatase activity. 
A H37RV SAESSAEKARDSEDRMRQFITDASHELRTPLTTIRGFAELYRQGAARDVGMLLSRIESEASRMGLLVDDLL H37Ra SAESSAEKARDSEDRMRQFITDASHELRTPLTTIRGFAELYRQGAARDVGMLLSRIESEASRMGLLVDDLL CS25 SAESSAEKARDSEDRMRQFITDASHELRTPLTTIRGFAELYRQGAARDVGMLLSRIESEASRMGLLVDDLL CS21 SAESSAEKARDSEDRMRQFITDASHELRTPLTTIRGFAELYRQGAARDVGMLLSRIESEASRMGLLVDDLL CS223 SAESSAEKARDSEDRMRQFITDASHELRTPLTTIRGFAELYRQGAARDVGMLLSRIESEASRMGLLVDDLL $* * * * * * * * * * * * * * * * * * * * * * * * * * * * * * * * * * * * * * * * * * * * * * * * * * * * * * * * * * * * * * * * * * * * * * *$

H37RV LLARLDAHRPLELCRVDLLALASDAAHDARAMDPKRRITLEVLDGPGTPEVLGDE SRLRQVLRNLVANAIQ H37Ra LLARLDAHRPLELCRVDLLALASDAAHDARAMDPKRRITLEVLDGPGTPEVLGDE SRLRQVLRNLVANAIQ CS25 LLARLDAHRPLELCRVDLLALASDAAHDARAMDPKRRITLEVLDGPGTPEVLGDE SRLRQVLRNLVANAIQ CS21 LLARLDAHRPLELCRVDLIALASDAAHDARAMDPKRRITLEVLDGPGTPEVLGDE SRLRQVLRNLVANAIQ CS223 LLARLDAHRPLELCRVDLLALASDAAHDARAMDPKRRITLEVLDGPGTPEVLGDE SRLRQVLRNLVANAIQ

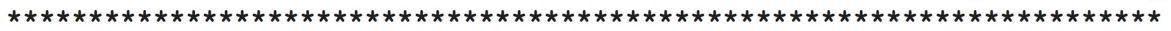

H37RV HTPESADVTVRVGTEGDDAILEVADDGPMSOEDALRVFERFYRADSSRARASGGTGLGLSIVDSLVAAHG H37Ra HTPESADVTVRVGTEGDDAILEYA DDGPGMSQEDALRVFERFYRADSSRARASGGTGLGLS IVDSLVAAHG CS25 HTPESADVTVRVGTEGDDAILEYTDDGPGSOEDALRVFERFYRADSSRARASGGTGLGLSIVDSLVAAHG CS21 HTPESADVTVRVGTEGDDAILEYTPDGPGMSQEDALRVFERFYRADSSRARASGGTGLGLSIVDSLVAAHG CS223 HTPESADVTVRVGTEGDDAILEYTPDGPGMSOEDALRVFERFYRADSSRARASGGTGLGLSIVDSLVAAHG

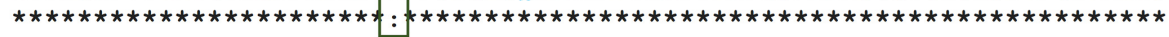

H37RV GAVTVTTALGEGCCFRVSLPRVSDVDQLSLTPVVPGPP H37Ra GAVTVTTALGEGCCFRVSLPRVSDVDQLSLTPVVPGPP CS25 GAVTVTTALGEGCCFRVSLPRVSDVDQLSLTPVVPGPP CS21 GAVTVTTALGEGCCFRVSLPRVSDVDQLSLTPVVPGPP

CS223 GAVTVTTALGEGCCFRVSLPRVSDVDQLSLTPVVPGPP

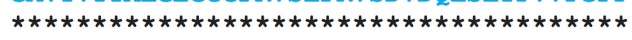

B

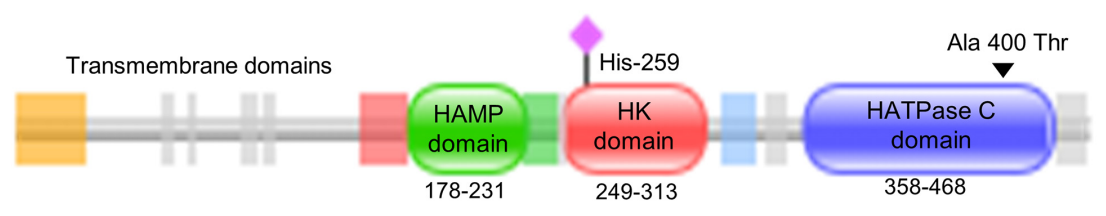

C

D

\begin{tabular}{|c|c|c|c|}
\hline \multirow{2}{*}{ Gene name } & \multicolumn{3}{|c|}{ Single Nucleotide Polymorphisms (SNPs) } \\
\cline { 2 - 4 } & $\begin{array}{c}\text { Total no. of } \\
\text { SNPs }\end{array}$ & Synonymous & $\begin{array}{c}\text { Non } \\
\text { synonymous }\end{array}$ \\
\hline PhoR (Rv0758) & 105 & 14 & 91 \\
\hline PhoP (Rv0757) & 11 & 6 & 5 \\
\hline DevS (Rv3132c) & 66 & 25 & 41 \\
\hline DevR (Rv3133c) & 12 & 6 & 6 \\
\hline DosT (Rv2027c) & 45 & 16 & 29 \\
\hline TcrY (Rv3764c) & 37 & 9 & 28 \\
\hline TcrX (Rv3765c) & 20 & 8 & 12 \\
\hline MtrB (Rv3245c) & 33 & 29 & 4 \\
\hline MtrA (Rv3246c) & 13 & 10 & 3 \\
\hline
\end{tabular}

\begin{tabular}{|c|c|c|}
\hline \multirow{2}{*}{$\begin{array}{c}\text { Examples of other } \\
\text { non synonymous } \\
\text { SNPs in PhoR }\end{array}$} & $\begin{array}{c}|c| \\
\text { GMTV } \\
\text { database }\end{array}$ & $\begin{array}{c}\text { PRJNA } \\
\text { 235851 } \\
\text { database }\end{array}$ \\
\cline { 2 - 3 } & $\begin{array}{c}0.0004 \% \\
(1 / 2501)\end{array}$ & $\begin{array}{c}0.5 \% \\
(2 / 242)\end{array}$ \\
\hline V105A & $\begin{array}{c}1 \% \\
(25 / 2501)\end{array}$ & Nil \\
\hline P106S & $\begin{array}{c}83.5 \% \\
(2089 / 2501)\end{array}$ & $\begin{array}{c}93 \% \\
(225 / 242)\end{array}$ \\
\hline P172L & $\begin{array}{c}1 \% \\
(27 / 2501)\end{array}$ & Nil \\
\hline V366G & \multicolumn{2}{|c}{} \\
\hline
\end{tabular}

FIGURE 1 | Single-nucleotide polymorphisms (SNPs) in the sensor kinase PhoR and other two-component systems (TCSs) in the clinical strains of Mycobacterium tuberculosis. (A) Sequence alignment of the sensor kinase (SK) protein PhoR, with H37Rv as the reference strain aligned with H37Ra and three clinical strains. HATPase domain is marked in blue with the mutation from alanine to threonine at 400 marked in green. CS25, CS21, and CS223 are the numbers assigned to three of the 223 clinical strains that carry this mutation analyzed in this study. (B) Pfam analysis of the SK protein PhoR from H37Rv, depicting the presence of three distinct domains, the HAMP domain (amino acids 178-231), the histidine kinase (HK) domain (amino acids 249-313), and the HATPase domain (amino acids 358-468). The predicted transmembrane domains are depicted to the left of these domains. The black triangle marks the mutation in the HATPase domain, and the pink diamond marks His ${ }^{259}$, the phosphorylation site. (C) Table showing the frequencies of synonymous and non-synonymous SNPs in various TCS proteins wrt H37Rv as reference genome reported in the GMTV database. (D) Table showing the frequencies of SNPs that are found in the PhoR sensor kinase in GMTV database and PRJNA235851 database. 
A

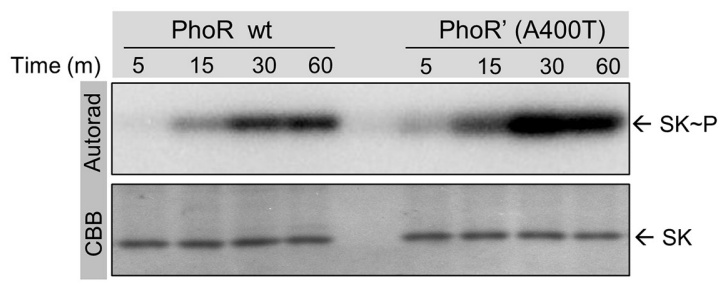

C

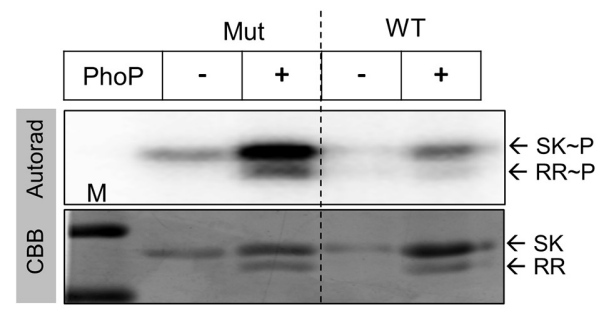

E

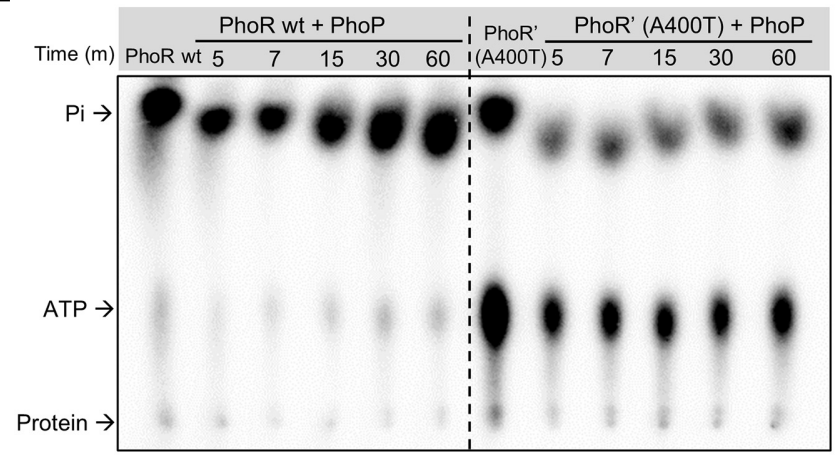

B

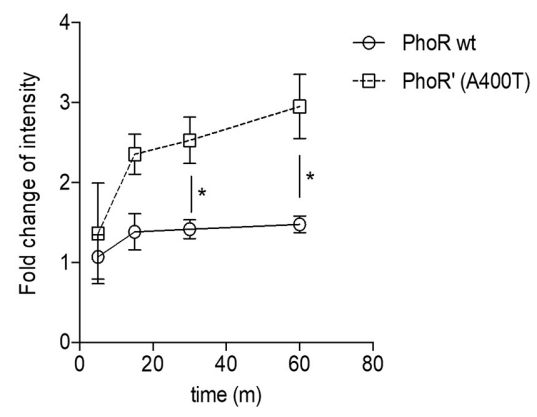

D

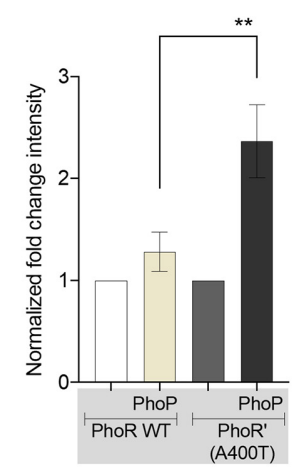

F

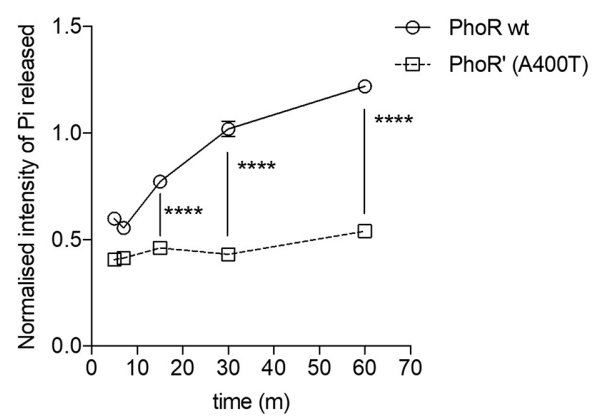

G

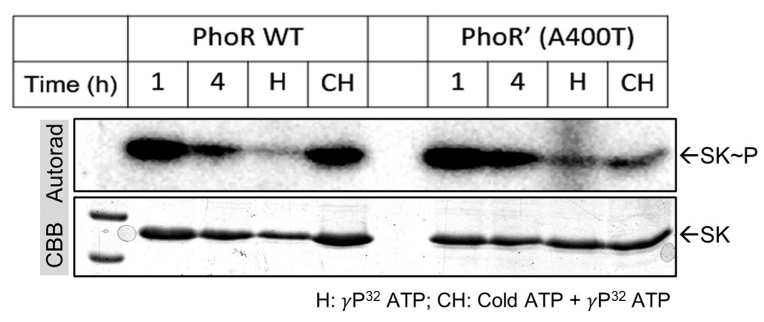

H

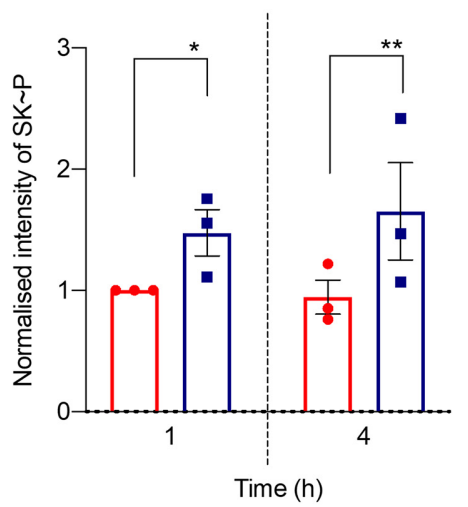

I

- PhoR wt P

- $P h o R^{\prime}(\mathrm{A} 400 \mathrm{~T}) \sim \mathrm{P}$

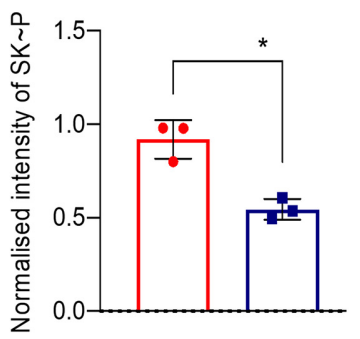

FIGURE 2 | Continued 

(SNP) on the biochemical activities of the sensor kinase PhoR.

(A) Autophosphorylation time-course analysis. The assay was performed using wild-type (left) and mutated PhoR' A400T (right) for indicated time points. Top panel, autoradiogram; bottom, Coomassie Brilliant Blue (CBB)-stained gel. (B) Quantitative measurement of autophosphorylated wild-type or mutated PhoR proteins at various time points as shown in panel (A). The signal recorded for both wild-type and mutant proteins at the first time point was taken as 1 , and the rest of the time points were normalized to it $(n=3)$. (C) Phosphotransfer analysis to analyze the effect of the mutation in the sensor kinase (SK) PhoR. The assay was performed using mutated (left) or wild-type PhoR (right) with wild-type response regulator (RR) protein PhoP. Top panel, autoradiogram; bottom panel, CBB-stained gel. (D) Quantitative analysis of signals of phosphorylated wild-type PhoR and mutated PhoR' A400T protein at 1-h post-phosphotransfer. The signal recorded for wild-type and mutant proteins without RR was taken as 1 , and the signal in the presence of $R R$ at various time points was normalized to it. (E) Thin-layer chromatography (TLC) analysis of phosphotransfer time-course reaction to determine the effect of the mutation on the phosphatase activity. The assay was performed using wild-type (left) or mutated PhoR' A400T (right) protein in the presence of the RR PhoP as described in "Materials and Methods" section. (F) Quantitative measurement of the amount of inorganic phosphate (Pi) generated by PhoR wt (represented by solid line, circles) or mutant PhoR (represented by dotted line, squares) in the presence of PhoP proteins at various time points as shown in panel (E). The Pi generated by the SK alone post $2 \mathrm{~h}$ of autophosphorylation was taken as 1 , and the subsequent time points are normalized to it $(n=3)$. (G) Kinase and phosphatase activity analysis of the PhoR proteins. The autophosphorylation assay was performed for indicated time points for wild-type (left) and mutant (right) PhoR protein, with conditions as indicated. Top panel, autoradiogram; bottom panel, Coomassie Brilliant Blue (CBB). Lanes marked 1 and 4, autophosphorylation reaction for 1 and $4 \mathrm{~h}$, respectively; lane $\mathrm{H}$, samples analyzed after heating at $95^{\circ} \mathrm{C}$ for $5 \mathrm{~min}$; and lane $\mathrm{HC}$, proteins first incubated with cold ATP for $2 \mathrm{~h}$ followed by ${ }^{32}$ P [ATP] for two additional hours before analysis (hot-chase analysis). (H) Quantitative analysis of autophosphorylation of wild-type and mutant PhoR proteins at 1- and 4-h incubation, as shown in panel (G). The signal from wild-type phosphorylated PhoR at $1 \mathrm{~h}$ is taken as 1 , and the rest of the points are normalized to it $(n=3)$. The signal intensity from the autorad was normalized to protein amount (as per CBB staining). (I) Quantitative analysis of SK phosphorylation post-incubation with cold ATP for $2 \mathrm{~h}$ followed by phosphorylation with ${ }^{32} \mathrm{P}$ labeled ATP for two additional hours (hot-chase analysis), as shown in panel (G), with lanes labeled $\mathrm{CH}$. The signals recorded for wild-type and mutant PhoR proteins were normalized to the amount of protein loaded $(n=3)$. The $p$-value was calculated based on the amount of SK P formed [for panels $(\mathbf{C}, \mathbf{E})]$ and the amount of Pi generated in the presence of the RR (PhoP) with respect to SK P [for panels (E,F)]. n represents the number of biological replicates used in the experiments. $p$-values, ${ }^{*} \leq 0.05,{ }^{* \star} \leq 0.01,{ }^{* \star \star} \leq 0.001$, and ${ }^{* \star \star *} \leq 0.0001$, were determined by two-way ANOVA for the autophosphorylation and time-course
FIGURE 2 | Analysis of the impact of A400T single-nucleotide polymorphism experiments and Student's $t$-tests for the phosphotransfer experiment.

$\mathrm{SK} \sim \mathrm{P}$. As expected, the mutant showed a higher signal than the WT, indicating that the mutant retains a higher phosphoryl signal.

In a parallel reaction aimed to examine phosphoryl group turnover, the SKs were first incubated with cold ATP for $2 \mathrm{~h}$, followed by the addition of ${ }^{32} \mathrm{P}$ labeled ATP for two additional hours (hot-chase analysis). This experiment's premise was that the protein that possesses higher ATP turnover would be labeled more with the ${ }^{32} \mathrm{P}$ in step 2 . In agreement with the observations above, we recorded higher labeling for WT PhoR protein, confirming that the mutant PhoR has a reduced autophosphatase activity (Figure 2G, lanes 4 and 9; and Figure 2I).

\section{PhoR' A400T Enhances the DNA-Binding Ability of RR PhoP on Target Gene aprA}

The phoPR TCS upon activation regulates close to $2 \%$ of M. tuberculosis's total genome, including a diverse set of genes involved in lipid synthesis, secretion of virulence factors, and genes regulating acid stress survival (14). apr genes form a part of the regulon of this TCS, and their expression increases at low pH when the PhoPR TCS is activated (Abramovitch et al., 2011).

The decreased phosphatase activity of the PhoR' (A400T) would effectively increase the availability of the phosphorylated kinase and consequently the activated RR PhoP. Thus, we examined the DNA-binding ability of phosphorylated PhoP generated through either PhoR wt or PhoR' (A400T) mutant proteins to the promoter region of aprA gene by EMSA. A 500bp region upstream of aprA gene labeled with $\gamma^{32} \mathrm{P}$-labeled ATP was used as a probe for these experiments. Although the RR alone at higher concentrations binds to the promoter, we used a lower optimized concentration $(1 \mu \mathrm{M})$, which did not show binding to the promoter in our assays (Figure 3A). When the probe was incubated with a mixture of SK and RR proteins in the presence of ATP, an increased mobility shift with increasing amounts of SK was recorded (Figure 3B). We also confirmed that the unphosphorylated SK, either WT or mutant, does not bind to the aprA promoter by themselves (Supplementary Figure 3). These findings suggest that an increase in autophosphorylation and phosphotransfer ability of the PhoR' (A400T) protein would induce higher downstream gene expression. This improved activation of the phoPR TCS regulon led us to examine changes brought about in vivo by this polymorphism.

To further test this, we analyzed the autophosphorylation of the WT and mutant PhoR under different conditions. First, we examined the phosphorylation over an extended period of $4 \mathrm{~h}$, and this revealed that, while $1 \mathrm{~h}$ of phosphorylation is sufficient to generate a peak signal for the proteins, significant phosphorylation was also detected at $4 \mathrm{~h}$; and as anticipated, it was higher in the mutant protein (Figure 2G, lanes 1 and 2 vs. lane 5 and 6; and Figure $2 \mathbf{H}$ ). Interestingly, heating of the reaction mix before analysis led to a significant phosphorylation reduction (Figure 2G, lanes 3 and 8). This could be due to the loss of SK-ATP complex [an intermediate formed during the autophosphorylation reaction, which yields SK P (Sankhe et al., 2018)], allowing signal only from

\section{In vivo Analysis of Downstream Gene Expression in Wild-Type or Mutant PhoR Complemented Strains of Mycobacterium tuberculosis H37Ra}

To check the mutant PhoR protein's effect in vivo, we cloned the phoPR operon from M. tuberculosis H37Rv with its native promoter ( $\sim 500$ bp upstream) in an integrative single-copy mycobacterial shuttle vector, pCV125. We introduced the mutation (A400T) in the WT construct and electroporated both the WT and mutant plasmids in H37Ra. We used H37Ra since it has a functionally defective PhoPR system and partially regains its virulence and persistence upon 
A

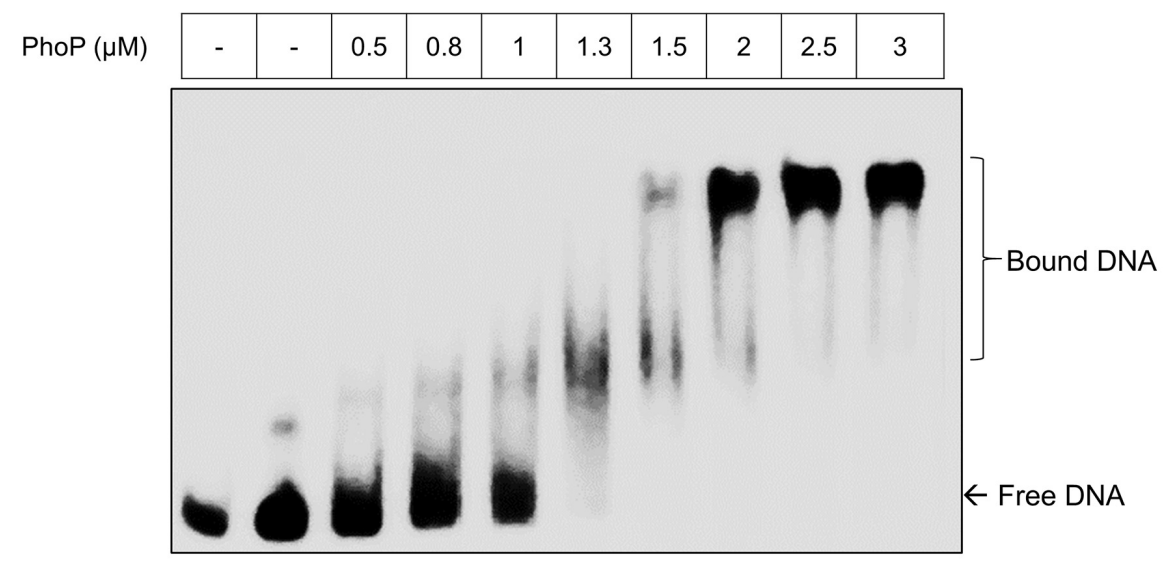

B

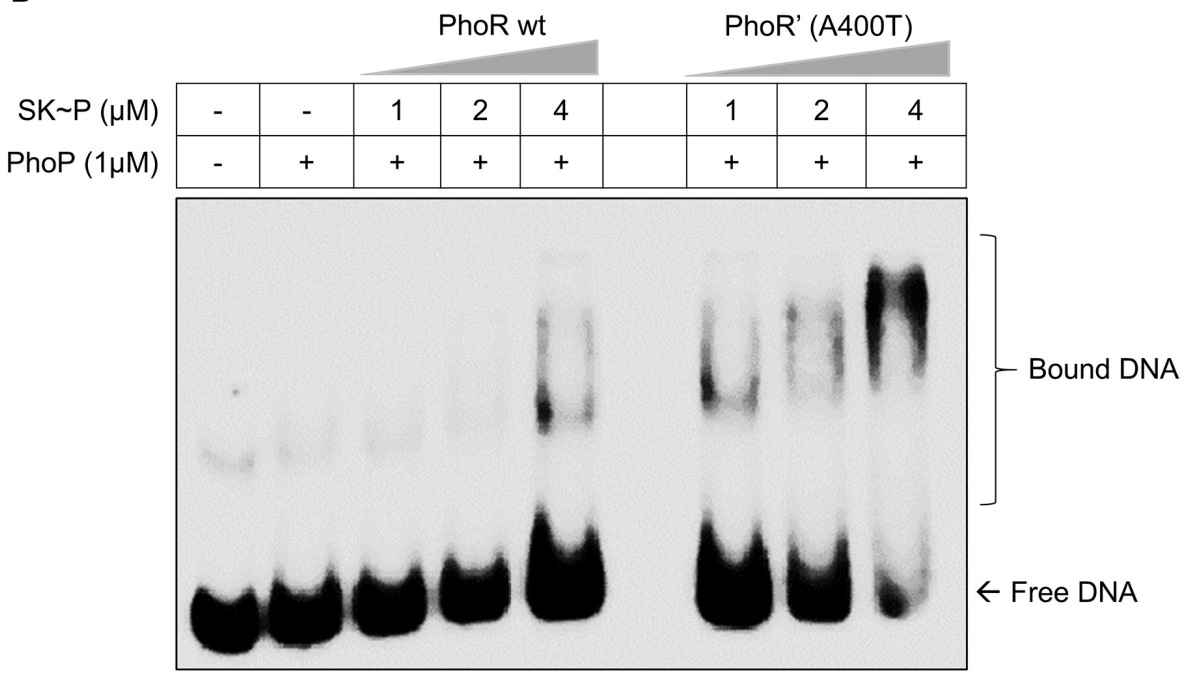

FIGURE 3 | Effect of the PhoR single-nucleotide polymorphism (SNP) on the DNA-binding activity of response regulator (RR) PhoP. (A) Titration of RR PhoP protein to determine a concentration that shows low binding of unphosphorylated PhoP to the promoter region of the aprA gene. The DNA fragment corresponding to the aprA promoter was incubated with an increasing concentration of PhoP protein (as indicated) and tested by electrophoretic mobility shift assay (EMSA). The RR

RegX3 was used as a negative control for non-specific binding (lane 2 marked -ve). (B) A comparative analysis of PhoP P (1 $\mu$ M) binding to aprA promoter DNA as a function of phosphorylation mediated by the wild-type [PhoR wt, left] or mutant [PhoR' (A400T), right] sensor kinase (SK) proteins at different concentrations. For all experiments, $n=3$, where $\mathrm{n}$ represents the number of biological replicates.

complementation with PhoP from H37Rv (Lee et al., 2008), making it a suitable model system for our studies. We checked the expression levels of phoR and phoP genes in the complemented strains by RT-PCR. Similar levels of $p h o R$ transcripts were observed in both strains under basal conditions at $\mathrm{pH}$ 7.2. At low $\mathrm{pH}$ (5.5), known to induce expression of phoPR TCS, we observed enhanced phoR expression in WT strain $(\sim 10$-fold $)$ as well as mutant strain $(\sim 12$-fold $)$ (Figure 4A). We also examined for change in the phoP transcript levels. As shown in Figure $4 \mathbf{B}$, phoP expression significantly increased in the mutant strain under inducing conditions $(\sim 8$-fold) as compared with a $\sim 1.5$-fold increase observed in the WT strain. Since no significant changes were observed with the mutant strain under uninduced conditions, we rationalized that the upregulation of $p h o P$ gene resulted from the increased phospho-signaling and downstream autoregulatory effect of the RR PhoP.

We also analyzed the expression levels of a specific downstream target gene, $p k s 2$ (polyketide synthase for SL-1 biosynthesis) (Walters et al., 2006). Expectedly, we observed a $\sim 10$-fold increase in $p k s 2$ transcripts in the mutant strain and a fourfold increase in the WT strain under inducing conditions (Figure 4C, left). These observations collectively validate the effect of an improved activation of the PhoP RR through phosphorylation by the PhoR' mutant SK on upregulation of PhoPR regulon (Figures $4 \mathrm{~A}-\mathrm{C}$, right). We also checked the 


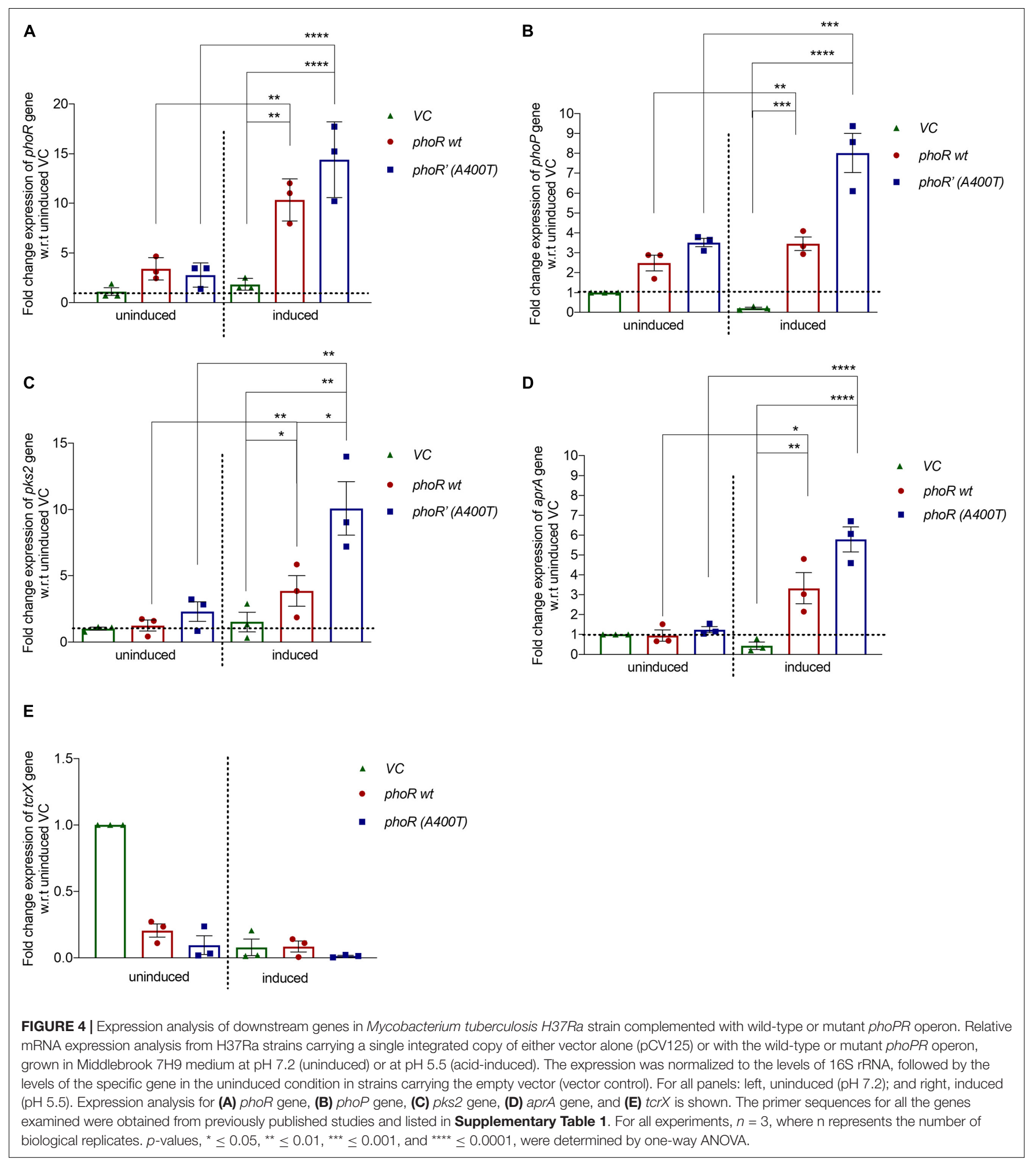

expression of aprA gene, another downstream gene of the PhoPR TCS, and found that it was induced under conditions of low $\mathrm{pH}$, as expected with the strains expressing the WT PhoR showing a $\sim 3$-fold induction and the mutant PhoR' strain sixfold induction relative to the vector control. More importantly, the induction was significantly higher (twofold) in mutant PhoR' carrying bacilli compared with the WT PhoR strains (Figure 4D, right); as a control, we analyzed the 


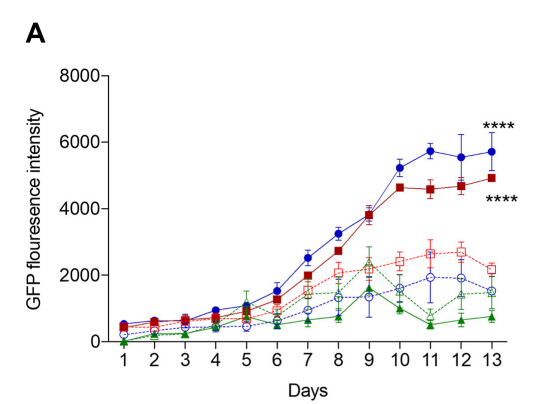

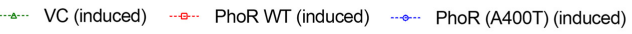

$\rightarrow$ VC (induced) $\rightarrow$ PhoR WT (induced) $\rightarrow$ PhoR (A400T) (induced)

C

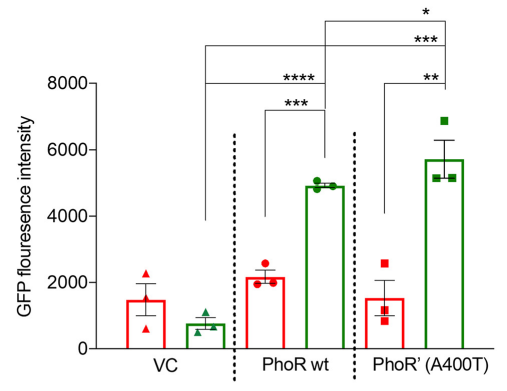

E
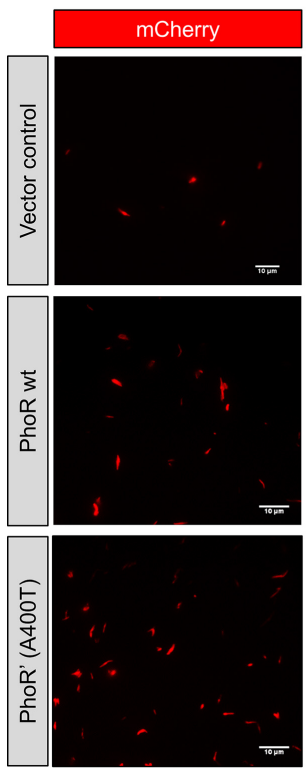

B

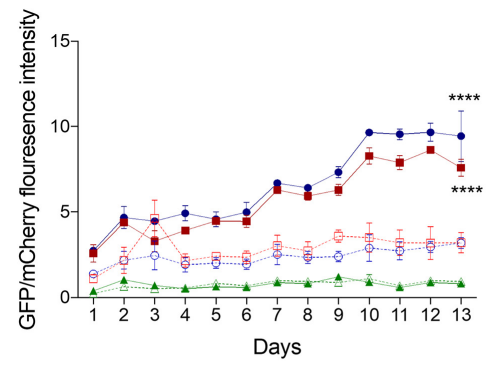

D

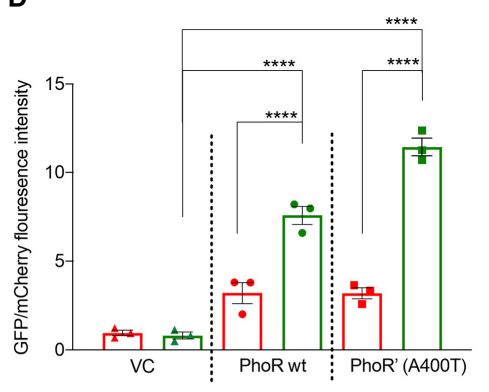

$\mathbf{F}$

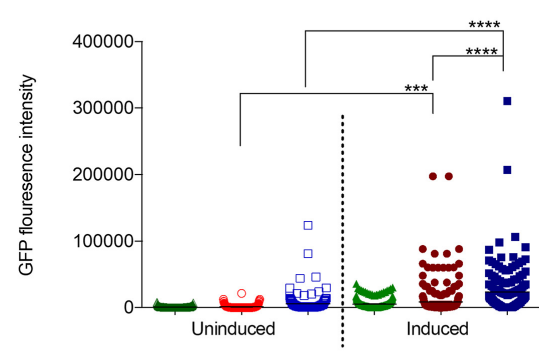

G

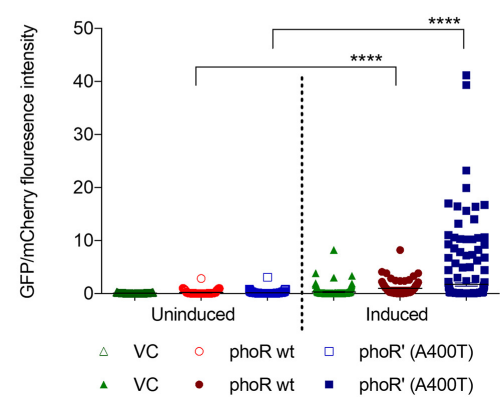

FIGURE 5 | Expression analysis of PhoPR regulated aprA gene using promoter-reporter system in Mycobacterium tuberculosis H37Ra. (A) Green fluorescent protein (GFP) fluorescence in H37Ra strains carrying aprA promoter-reporter plasmid and integrative vector pCV125 alone or containing wild-type or mutant phoPR operon in cultures grown at pH 7.2 or at pH 5.5. The solid lines show GFP fluorescence at pH 5.5, and the dotted lines that at pH 7.2 over 13 days. (B) Analysis of the relative fluorescence ratio of GFP/mCherry, at pH 5.5, solid lines; and at pH 7.2, dotted lines (triangles, vector control; circles, phoR wt; and squares, phoR' (A400T) mutant). (C) Quantitation of GFP fluorescence in cultures (as indicated) at day 12 at pH 5.5 (green, induced) and at pH 7.2 (red, uninduced). (D) Quantitation of the relative fluorescence ratio of GFP/mCherry from induced and uninduced cultures at day 12 at pH 5.5 (green) and at pH 7.2 (red). (E) Fluorescence microscopy images showing GFP expression in cultures at day 12 in indicated strains. Left panel, mCherry; right panel, GFP. (F) Quantitation of GFP fluorescence and (G) relative fluorescence by GFP/mCherry ratios by fluorescence microscopy in various strains and conditions (as indicated) from $\sim 300$ individual bacilli. $n=3$, where $n$ represents the number of biological replicates used in the experiments. $p$-values ${ }^{*} \leq 0.05,{ }^{* *} \leq 0.01,{ }^{* \star *} \leq 0.001$, and ${ }^{* \star * *} \leq 0.0001$, were determined by two-way ANOVA. 
A

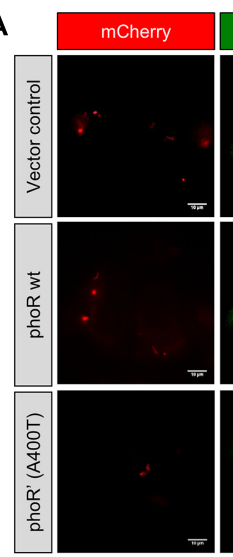

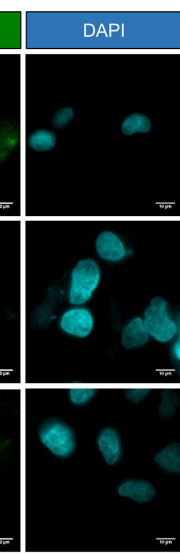

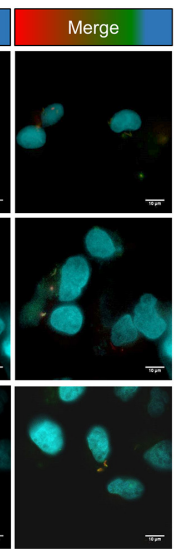

B

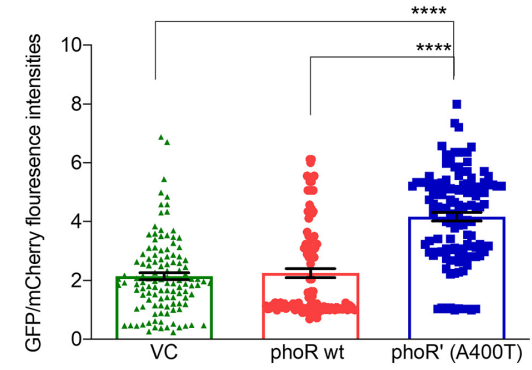

C

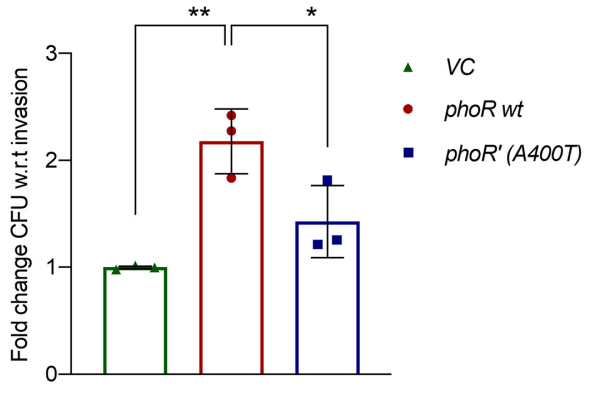

D

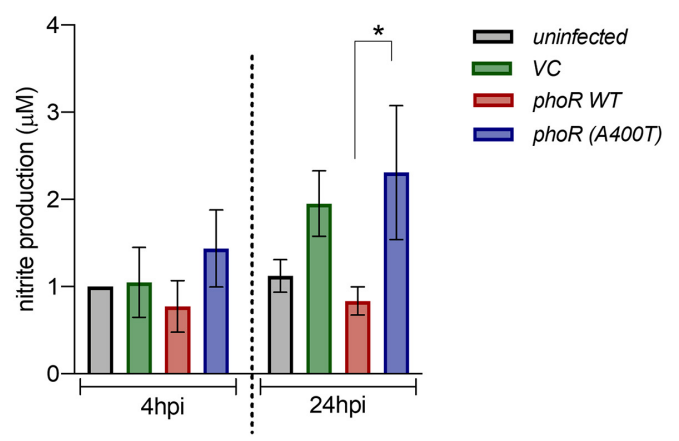

FIGURE 6 | Analysis of the effect of the phoR polymorphism by infection studies in host cells using H37Ra strain as a host strain. (A) Fluorescence microscopy images showing aprA induction upon infection in $\mathrm{H} 460$ lung epithelial cell line at $4 \mathrm{hpi}$. Top panel, a strain carrying vector alone; middle panel, PhoR wt; and lower panel, PhoR' (A400T) mutant. mCherry marks constitutive promoter activity; green fluorescent protein (GFP), aprA marks promoter activity; DAPI marks the nucleus of the host cell; and merged image shows localization for all fluorophores. The brightness of merged image was increased to show presence of bacillary signal. (B) Quantification of relative fluorescence $4 \mathrm{hpi}$ of intracellular bacilli by GFP/mCherry ratio for 100 bacilli. Cells were infected with H37Ra strains carrying aprA promoter-reporter plasmid along with an integrated copy of the empty vector, or vector containing either the wild-type or mutant phoPR operon. (C) Quantitation of the intracellular bacterial burden for various strains (as indicated) after infection in THP-1 differential macrophage cell line. Cells were infected with H37Ra strains an integrated copy of the empty vector, the wild-type or mutant phoPR operon. Colony-forming units (CFUs) obtained at 24 hpi are shown. (D) Measurement of NO production in supernatants of uninfected and infected THP-1 cells by the Griess test at various time points. Cells were infected with strains described above, and the $\mathrm{NO} /$ nitrite levels at $4 \mathrm{hpi}$ were measured; left panel, $24 \mathrm{hpi}$; right panel, $72 \mathrm{hpi}$. The nitrite produced is normalized to the uninfected cells at $4 \mathrm{hpi}$. For all experiments, $n=3$, where $n$ represents the number of biological replicates used in the experiments. $p$-values, ${ }^{*} \leq 0.05,{ }^{* \star} \leq 0.01,{ }^{\star * *} \leq 0.001$, and ${ }^{* \star \star *} \leq 0.0001$, were determined by one-way ANOVA.

expression of $R v 3764$ ( $t c r X$ ) gene and found no significant change in its expression levels among the strains in any condition (Figure 4E).

\section{Expression of aprA Is Induced to a Greater Extent in PhoR' Mutant Strain at Acidic pH}

Given that we found differences in the induction and expression of Pho regulon in the WT and mutant strains in response to $\mathrm{pH}$ stimulation, we introduced a promoterreporter plasmid containing aprA promoter-driven eGFP2 and constitutively expressed mCherry (Abramovitch et al., 2011). The objective was to measure GFP fluorescence as a readout of the activation of the TCS. The strains carrying WT or mutant PhoPR showed induction of an aprA promoter activity over 12 days at acidic $\mathrm{pH}$ of 5.5 , unlike at $\mathrm{pH} 7.2$ (Figure 5A). A 12-day time point was chosen, as it has been previously reported to be reliable time point to record acidmediated induction (Abramovitch et al., 2011). As anticipated, the strain-carrying mutant PhoPR TCS demonstrated a higher expression of aprA GFP over time, with a significant difference at day 12 compared with the strains with WT PhoPR and the vector control (Figure 5C). When normalized to mCherry, we observed a significantly higher expression of aprA in the mutant than the WT (Figures 5B,D). A similar observation of expression differences in these strains at day 12 post-acid induction was made by microscopic analysis (Figure 5E). Quantification of fluorescence from $\sim 300$ bacterial cells expressing both GFP and mCherry per strain (Figures 5F,G) confirmed that the mutant strain has a higher aprA promoter activity. 
To verify that the activation of aprA at low $\mathrm{pH}$ is through the phoPR TCS, we used an inhibitor of this TCS ETZ, previously reported to inhibit the phoPR system (Johnson et al., 2015). After $24 \mathrm{~h}$ of treatment with $80 \mu \mathrm{M}$ of ETZ (Supplementary Figure 4A) at day 12 post-induction, reduction in the expression of aprA fluorescence was recorded (Supplementary Figures 5A,B) in the strain carrying the WT copy. However, the reduction was lesser in the strain complemented with mutant PhoR, showing that the SNP in clinical strains could potentially alter their response to drugs. To determine where this inhibitor could be acting, we checked the autophosphorylation of PhoR wt and PhoR' (A400T) protein in the presence of different concentrations of the inhibitor. We observed inhibition of phosphorylation for both WT and mutant SK proteins. This inhibition was lesser in the mutant than the WT (Supplementary Figures 4C,D), suggesting that the inhibition of the TCS PhoPR observed by previous researchers and us could be by lowering the SK activation levels by yetexplored mechanisms.

\section{Effect of the Single-Nucleotide Polymorphism (A400T) in PhoR on Intracellular Growth of Complemented Mycobacterium tuberculosis H37Ra Strain in Host Cells}

Having established that the SNP alters expression of PhoPregulated genes in vivo, by quantifying the aprA reporter activity at various time points post-infection ( $4 \mathrm{hpi}$ ), we found that the expression of aprA-GFP was persistently higher in the mutant strain, and hence quantified the promoter activity for $\sim 100$ bacilli of each strain inside the infected H460 lung epithelial cell line (Figures 6A,B). We also performed these experiments in the presence of the inhibitor ETZ at a concentration of $100 \mu \mathrm{M}$. This concentration does not affect the host cells' health or the bacteria (Supplementary Figure 4B). We recorded differences in the expression of aprA-GFP, with the mutant showing higher expression of aprA-GFP at any given time point (Figure 6B). We also investigated the ability of the mutant strain to survive within differentiated THP-1 cells, with the three test strains of H37Ra carrying the pCV125 empty vector, the pho $R$ WT, or phoR' (A400T) at an MOI of 10 and plated for colonyforming unit (CFU) differences at $24 \mathrm{hpi}$ and normalized to the invasion time point at $4 \mathrm{hpi}$ (Figure 6C). Interestingly, while the complementation with the WT PhoR increased the CFU by more than twofold, the presence of mutant PhoR only showed marginal increase in the bacterial load, suggesting an impaired survival of the strain, similar to vector alone (Figure 6C). Thus, we hypothesize that inefficient survival within cells may be a key factor for this SNP not being selected naturally in the population.

The survival advantage of the strain carrying the WT PhoPR protein could plausibly be driven by alteration in host responses such as cytokine levels and the oxidative burst (Voskuil et al., 2011). We examined if the host responds differently to the WT and mutant strains, by monitoring the NO levels from infected THP-1 cells at various time points ( 4 and $24 \mathrm{hpi}$ ) using the
Griess reagent. In agreement with previously published data (41), the NO levels in the cells infected with the WT PhoR strain were always lower owing to an active $p h o P R$ regulon of H37Rv (Figure 6D; Ferrer et al., 2010), which is reflected in the higher CFU load obtained from them. Interestingly, the production of NO when infected with the strain containing mutant PhoR' (A400T) was $\sim 1.5$-fold higher than the WT and similar to the strain containing the empty vector and at $24 \mathrm{hpi}$ (Figure 6D, right panel). This response was captured on the bacterial burden as well (Figure 6C), and both vector control and mutant PhoR strains had similar CFU, which was lower than that of WT PhoR. Thus, the host response could be a significant contributor to the absence of selection of this mutation in clinical strains, as the increase in NO could be one of the ways the host eradicates the mutant strain more effectively. These observations reveal that multiple factors drive infection and survival in the host, such as the adaptivity of the strain to infect and survive in the host and highlight the finding that the PhoR polymorphism at A400T position, which though robustly activates the phoPR TCS and downstream operon activity, is not favorable for invasion and survival conditions in vivo.

Overall, we present experimental evidences underlying the occurrence of a poorly prevalent polymorphism of PhoR SK in clinical strains, which improves its biochemical activities but reduces its overall fitness by affecting its ability to invade and survive in the intracellular environment in vitro.

\section{DISCUSSION}

Various reports on genetic variations among strains of M. tuberculosis used in the laboratory and clinical strains address the diversity that exists among them (Ramaswamy and Musser, 1998; Fleischmann et al., 2002; Zheng et al., 2008; Schreuder et al., 2015). However, these reports seldom address the variations seen in the TCS regions of clinical strains nor addresses their effects on signaling. We report and characterize a rare polymorphism in the PhoR SK protein that establishes an altered signaling landscape in the tubercle bacteria.

The PhoPR TCS is relatively well-studied in M. tuberculosis and implicated in regulating many essential processes such as virulence, immunogenicity, persistence, lipid synthesis and metabolism, cell wall composition, and aerobic and anaerobic respiration (Pérez et al., 2001; Gupta et al., 2006; Walters et al., 2006; Gonzalo-Asensio et al., 2008; Pathak et al., 2010). The PhoR histidine SK protein has an integral membraneanchored domain, with an extracellular domain to sense extracellular cues (Ryndak et al., 2008) and a typical kinase domain containing the SHELR motif with the conserved histidine residue as phosphorylation site. The mutation A400T, which maps in the HATPase domain of the protein, enhances its catalytic activity in terms of stable phosphotransfer and reduced autophosphatase activity, which is rarely examined, ultimately affecting the quantum of the activated RR PhoP available in the system. The mutation abrogated the rapid decay of phosphorylated SK and RR, which we generally record for the 
PhoPR system, thereby facilitating more persistent activation of the PhoPR regulon. This change enhanced the levels of PhoP $\sim \mathrm{P}$, leading to enhancement in its DNA binding to target gene promoters, such as for the aprA (acid and phagosome regulated) locus. These changes when tested in vivo in acidic $\mathrm{pH}$ revealed robust activation of the $\mathrm{H}^{+}$sensing TCS, PhoPR in the mutant strain.

For our studies, we used an avirulent mycobacterial strain, H37Ra, which has a mutation that impairs the DNA-binding activity of the PhoP protein (Chesne-Seck et al., 2008; Zheng et al., 2008). The strain offered us an advantage, as the mutation's effect can be recorded without generating a knockout strain. The impact of the SK protein's altered activity in the A400T mutant is still profoundly seen at the levels of downstream genes, highlighting the utility of H37Ra in such studies.

Besides $\operatorname{apr} A$, expression analysis of the $p h o R, p h o P$, and $p k s 2$, other downstream genes, showed higher levels in the strains carrying WT or mutant phoR gene, unlike one carrying the vector only. When the expression was analyzed by growing the cultures at a $\mathrm{pH}$ of 5.5 over 12 days, an induction was recorded for all of them, proving that the complementation of phoPR into $\mathrm{H} 37 \mathrm{Ra}$ reconstitutes the functional PhoPR operon; however, there was no induction of another TCS gene tcrX when tested. However, a significantly higher expression was observed for the mutant PhoR strain due to its enhanced kinase activity and impaired phosphatase activity. In the functional complementation background, the readout from aprA promoterreporter plasmid (Abramovitch et al., 2011) showed a higher expression in the presence of mutant PhoR compared with the WT PhoR and the vector control, thus indirectly providing evidence that low $\mathrm{pH}$ is linked to activation of PhoR, enhancing the kinase activity of the PhoR protein. This aspect has not been established to date owing to the lack of biochemically characterized full-length proteins.

Based on the experimental evidence, it was tempting to hypothesize that this improved response to acidic $\mathrm{pH}$ could benefit the mutant strain. However, in infection studies, we recorded lower bacterial burden in the mutant strain, compared with the strain carrying WT PhoPR locus. This observation correlated well with the changes in the NO levels in the infected cells, where the WT showed reduced NO levels and higher bacterial burden.

Our results display the importance and selection of a regulated and evolutionarily tuned signaling cascade during infection. Even though the polymorphism seems to provide a functional advantage and primes the $p h o P R$ operon for environmental stimuli, early and higher expression of the target genes is not favorable for the bacteria during infection. Similar observations have been made for several viral infections and mutations with a low invasion profile and are not very successful. For instance, HIV-1 subtype C accounts for over $50 \%$ of the conditions globally. However, it is less fit within-host than the less prevalent HIV-1 subtype B (Shet et al., 2016), possibly because of its relatively improved ability to be transmitted successfully to new hosts (Ariën et al., 2007).

An exciting offshoot of our study is the implication of SNPs on drug resistance. In an attempt to ensure that PhoPR is the

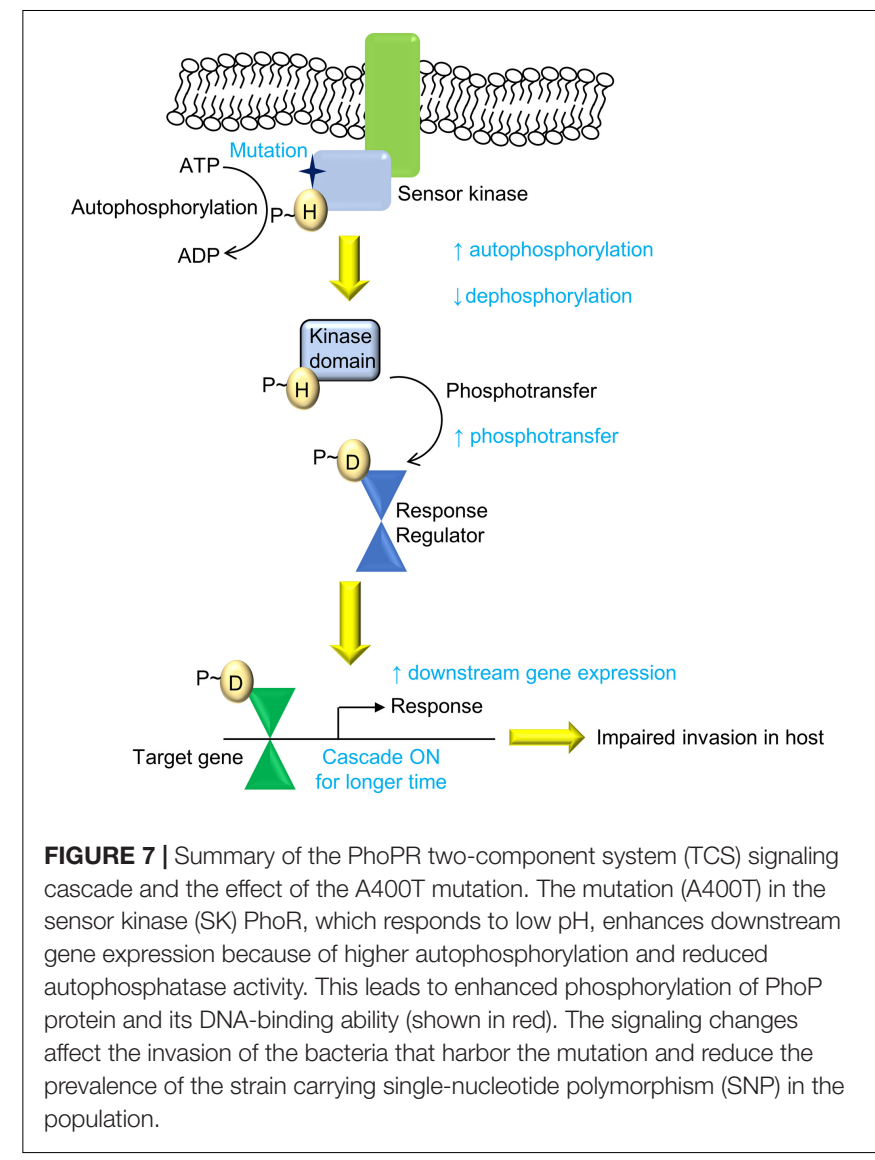

primary regulator of our observations under low $\mathrm{pH}$, we used an inhibitor ETZ, previously reported to inhibit acid sensing in this system. While the inhibitor lowered the expression of target gene aprA and reduced CFU in cell line infection, this inhibitor when tested in the autophosphorylation assay showed reduced inhibitory activity against the mutant protein. This observation suggests the importance of characterizing the effect of inhibitors vis-à-vis polymorphisms seen in the target protein in clinical strains, which would affect the efficacy of various new therapeutic entities.

Overall, we present an evolutionary design of signaling systems in bacteria, wherein genetic polymorphisms that can bring about an increase in signaling may not be favorable for the bacteria and hence may be deselected. Here, the mutation in the SK PhoR had better activity but limited invasion ability, possibly through altered host responses upon infection (Figure 7), facilitating its clearance through processes such as the production NO, which limits its replication within the host. Though the mechanisms by which this is governed are yet to be elucidated, such changes confer increased pathogenesis but impaired transmission in clinical strains such as in M. tuberculosis IS6110 B strain (Soto et al., 2004; Broset et al., 2015). Our finding supports the understanding that the balance between gene expression levels and their temporal dynamics needs to be optimized to maintain pathogenicity and virulence. 


\section{DATA AVAILABILITY STATEMENT}

The original contributions presented in the study are included in the article/ Supplementary Material, further inquiries can be directed to the corresponding author/s.

\section{AUTHOR CONTRIBUTIONS}

UW designed the study, performed the experiments, analyzed the data, and wrote the manuscript. MK performed project administration and supervision. VM analyzed the data and wrote the manuscript. ND provided conceptualization and project administration. DS conceived the study, analyzed the data, and wrote the manuscript. All authors contributed to the article and approved the submitted version.

\section{FUNDING}

This study was supported by the Department of Science and Technology, INSPIRE scholarship to UW (IF150491) and Department of Biotechnology, India (grant nos. BT/PR17357/MED/29/1019/2016 and

\section{REFERENCES}

Abramovitch, R. B., Rohde, K. H., Hsu, F. F., and Russell, D. G. (2011). aprABC: a Mycobacterium tuberculosis complex-specific locus that modulates $\mathrm{pH}$-driven adaptation to the macrophage phagosome. Mol. Microbiol. 80, 678-694. doi: 10.1111/j.1365-2958.2011.07601.x

Agrawal, R., Pandey, A., Rajankar, M. P., Dixit, N. M., and Saini, D. K. (2015). The two-component signalling networks of Mycobacterium tuberculosis display extensive cross-talk in vitro. Biochem. J. 469, 121-134. doi: 10.1042/BJ20150268

Ariën, K. K., Vanham, G., and Arts, E. J. (2007). Is HIV-1 evolving to a less virulent form in humans? Nat. Rev. Microbiol. 5, 141-151. doi: 10.1038/nrmicro1594

Borrell, S., Trauner, A., Brites, D., Rigouts, L., Loiseau, C., Coscolla, M., et al. (2019). Reference set of Mycobacterium tuberculosis clinical strains: a tool for research and product development. PLoS One 14:e0214088. doi: 10.1371/journal.pone. 0214088

Bourret, R. B., and Silversmith, R. E. (2010). Two-component signal transduction. Curr. Opin. Microbiol. 13, 113-115. doi: 10.1016/j.mib.2010.02.003

Broset, E., Martín, C., and Gonzalo-Asensio, J. (2015). Evolutionary landscape of the Mycobacterium tuberculosis complex from the viewpoint of phoPR: implications for virulence regulation and application to vaccine development. mBio 6:e01289-15. doi: 10.1128/mBio.01289-1215

Chai, Q., Zhang, Y., and Liu, C. H. (2018). Mycobacterium tuberculosis: an adaptable pathogen associated with multiple human diseases. Front. Cell. Infect. Microbiol. 8:158. doi: 10.3389/fcimb.2018.00158

Chernyaeva, E. N., Shulgina, M. V., Rotkevich, M. S., Dobrynin, P. V., Simonov, S. A., Shitikov, E. A., et al. (2014). Genome-wide Mycobacterium tuberculosis variation (GMTV) database: a new tool for integrating sequence variations and epidemiology. BMC Genomics 15:308. doi: 10.1186/1471-2164-15-308

Chesne-Seck, M. L., Barilone, N., Boudou, F., Asensio, J. G., Kolattukudy, P. E., Martín, C., et al. (2008). A point mutation in the two-component regulator PhoP-PhoR accounts for the absence of polyketide-derived acyltrehaloses but not that of phthiocerol dimycocerosates in Mycobacterium tuberculosis H37Ra. J. Bacteriol. 190, 1329-1334. doi: 10.1128/JB.01465-1467

Cimino, M., Thomas, C., Namouchi, A., Dubrac, S., Gicquel, B., and Gopaul, D. N. (2012). Identification of DNA binding motifs of the Mycobacterium tuberculosis phop/phor two-component signal transduction system. PLoS One 7:e42876. doi: 10.1371 /journal.pone.0042876
BIRAC/PMU/2019/Sentinels-001) to DS. The study is also supported in part by the DBT partnership program to Indian Institute of Science (BT/PR27952-INF/22/212/2018) and Infosys Foundation; equipment support was provided by DSTFunds for Infrastructure in Science and Technology program (SR/FST/LSII-036/2016) and University Grants Commission [F.4.13//DRS-III (SAP-II)].

\section{ACKNOWLEDGMENTS}

We would like to thank Sheryl E. Fernandes for RT-PCR analysis; Krishna Kumar Singh and Devendra Pratap Singh for critical feedback; Ashlee M. Earl, Broad Institute, for guidance on accessing strain sequences on NCBI projects; and Robert Abramovitch for aprA promoter-reporter system and IISc for various resources.

\section{SUPPLEMENTARY MATERIAL}

The Supplementary Material for this article can be found online at: https://www.frontiersin.org/articles/10.3389/fmicb. 2021.724482/full\#supplementary-material

Cole, S. T., Brosch, R., Parkhill, J., Garnier, T., Churcher, C., Harris, D., et al. (1998). Deciphering the biology of Mycobacterium tuberculosis from the complete genome sequence. Nature 396, 190-190. doi: 10.1038/24206

Comas, I., Coscolla, M., Luo, T., Borrell, S., Holt, K. E., Parkhill, J., et al. (2014). Mycobacterium tuberculosis with modern humans. Nat. Genet. 45, 1176-1182. doi: 10.1038/ng.2744.Out-of-Africa

Coscolla, M., and Gagneux, S. (2014). Consequences of genomic diversity in Mycobacterium tuberculosis. Semin. Immunol. 26, 431-444. doi: 10.1016/j. smim.2014.09.012

Dubey, B. N., Lori, C., Ozaki, S., Fucile, G., Plaza-Menacho, I., Jenal, U., et al. (2016). Cyclic di-GMP mediates a histidine kinase/phosphatase switch by noncovalent domain cross-linking. Sci. Adv. 2:e1600823. doi: 10.1126/sciadv. 1600823

Estrella, J. L., Kan-Sutton, C., Gong, X., Rajagopalan, M., Lewis, D. E., Hunter, R. L., et al. (2011). A novel in vitro human macrophage model to study the persistence of Mycobacterium tuberculosis using vitamin D3 and retinoic acid activated THP-1 macrophages. Front. Microbiol. 2:67. doi: 10.3389/fmicb.2011.0 0067

Ferrer, N. L., Gomez, A. B., Neyrolles, O., Gicquel, B., and Martin, C. (2010). Interactions of attenuated Mycobacterium tuberculosis phoP mutant with human macrophages. PLoS One 5:e12978. doi: 10.1371/journal.pone.0012978

Fleischmann, R. D., Alland, D., Eisen, J. A., Carpenter, L., White, O., Peterson, J., et al. (2002). Whole-Genome comparison of. Society 184, 5479-5490. doi: 10.1128/JB.184.19.5479

Garnier, T., Eiglmeier, K., Camus, J. C., Medina, N., Mansoor, H., Pryor, M., et al. (2003). The complete genome sequence of Mycobacterium bovis. Proc. Natl. Acad. Sci. U S A. 100, 7877-7882. doi: 10.1073/pnas.1130426100

Gonzalo-Asensio, J., Malaga, W., Pawlik, A., Astarie-Dequeker, C., Passemar, C., Moreau, F., et al. (2014). Evolutionary history of tuberculosis shaped by conserved mutations in the PhoPR virulence regulator. Proc. Natl. Acad. Sci. U S A. 111, 11491-11496. doi: 10.1073/pnas.1406693111

Gonzalo-Asensio, J., Mostowy, S., Harders-Westerveen, J., Huygen, K., HernándezPando, R., Thole, J., et al. (2008). PhoP: a missing piece in the intricate puzzle of Mycobacterium tuberculosis virulence. PLoS One 3:e3496. doi: 10.1371/journal. pone.0003496

Gupta, S., Sinha, A., and Sarkar, D. (2006). Transcriptional autoregulation by Mycobacterium tuberculosis PhoP involves recognition of novel direct repeat 
sequences in the regulatory region of the promoter. FEBS Lett. 580, 5328-5338. doi: 10.1016/j.febslet.2006.09.004

Homolka, S., Niemann, S., Russell, D. G., and Rohde, K. H. (2010). Functional genetic diversity among Mycobacterium tuberculosis complex clinical isolates: delineation of conserved core and lineage-specific transcriptomes during intracellular survival. PLoS Pathog. 6:e1000988. doi: 10.1371/journal.ppat. 1000988

Ioerger, T. R., Feng, Y., Ganesula, K., Chen, X., Dobos, K. M., Fortune, S., et al. (2010). Variation among genome sequences of H37Rv strains of Mycobacterium tuberculosis from multiple laboratories. J. Bacteriol. 192, 3645-3653. doi: 10. 1128/JB.00166- 110

Johnson, B. K., Colvin, C. J., Needle, D. B., Mba Medie, F., Champion, P. A. D. G., and Abramovitch, R. B. (2015). The carbonic anhydrase inhibitor ethoxzolamide inhibits the Mycobacterium tuberculosis PhoPR regulon and Esx-1 secretion and attenuates virulence. Antimicrob. Agents Chemother. 59, 4436-4445. doi: 10.1128/AAC.00719-715

Lee, J. S., Krause, R., Schreiber, J., Mollenkopf, H. J., Kowall, J., Stein, R., et al. (2008). Mutation in the transcriptional regulator phop contributes to avirulence of Mycobacterium tuberculosis H37Ra strain. Cell Host Microbe 3, 97-103. doi: 10.1016/j.chom.2008.01.002

Liu, F., Hu, Y., Wang, Q., Li, H. M., Gao, G. F., Liu, C. H., et al. (2014). Comparative genomic analysis of Mycobacterium tuberculosis clinical isolates. BMC Genomics 15:469. doi: 10.1186/1471-2164-15-469

Ludwiczak, P., Gilleron, M., Bordat, Y., Martin, C., Gicquel, B., and Puzo, G. (2002). Mycobacterium tuberculosis phoP mutant: lipoarabinomannan molecular structure. Microbiology 148, 3029-3037. doi: 10.1099/00221287-14810-3029

Madeira, F., Park, Y. M., Lee, J., Buso, N., Gur, T., Madhusoodanan, N., et al. (2019). The EMBL-EBI search and sequence analysis tools APIs in 2019. Nucleic Acids Res. 47, W636-W641. doi: 10.1093/nar/gkz268

Manson, A. L., Abeel, T., Galagan, J. E., Sundaramurthi, J. C., Salazar, A., Gehrmann, T., et al. (2017). Mycobacterium tuberculosis whole genome sequences from Southern India suggest novel resistance mechanisms and the need for region-specific diagnostics. Clin. Infect. Dis. 64, 1494-1501. doi: 10. 1093/cid/cix169

Mohan, A., Padiadpu, J., Baloni, P., and Chandra, N. (2015). Complete genome sequences of a Mycobacterium smegmatis laboratory. Genome Announc. 3, 4-5. doi: 10.1128/genomeA.01520-14

Pang, X., Vu, P., Byrd, T. F., Ghanny, S., Soteropoulos, P., Mukamolova, G. V., et al. (2007). Evidence for complex interactions of stress-associated regulons in an mprAB deletion mutant of Mycobacterium tuberculosis. Microbiology 153, 1229-1242. doi: 10.1099/mic.0.29281-29280

Pathak, A., Goyal, R., Sinha, A., and Sarkar, D. (2010). Domain structure of virulence-associated response regulator Phop of Mycobacterium tuberculosis: role of the linker region in regulator-promoter interaction(s). J. Biol. Chem. 285, 34309-34318. doi: 10.1074/jbc.M110.135822

Pérez, E., Samper, S., Bordas, Y., Guilhot, C., Gicquel, B., and Martín, C. (2001). An essential role for phoP in Mycobacterium tuberculosis virelence. Mol. Microbiol. 41, 179-187. doi: 10.1046/j.1365-2958.2001.02500.x

Ramaiah, A., Nayak, S., Rakshit, S., Manson, A. L., Abeel, T., Shanmugam, S., et al. (2019). Evidence for highly variable, region-specific patterns of T-cell epitope mutations accumulating in mycobacterium tuberculosis strains. Front. Immunol. 10:195. doi: 10.3389/fimmu.2019.00195

Ramaswamy, S., and Musser, J. M. (1998). Molecular genetic basis of antimicrobial agent resistance in Mycobacterium tuberculosis: 1998 update. Tuber. Lung Dis. 79, 3-29. doi: 10.1054/tuld.1998.0002

Rose, G., Cortes, T., Comas, I., Coscolla, M., Gagneux, S., and Young, D. B. (2013). Mapping of genotype-phenotype diversity among clinical isolates of Mycobacterium tuberculosis by sequence-based transcriptional profiling. Genome Biol. Evol. 5, 1849-1862. doi: 10.1093/gbe/evt138

Rouse, D. A., Li, Z., Bai, G. H., and Morris, S. L. (1995). Characterization of the katG and inhA genes of isoniazid-resistant clinical isolates of Mycobacterium tuberculosis. Antimicrob. Agents Chemother. 39, 2472-2477. doi: 10.1128/AAC. 39.11.2472

Ryndak, M., Wang, S., and Smith, I. (2008). PhoP, a key player in Mycobacterium tuberculosis virulence. Trends Microbiol. 16, 528-534. doi: 10.1016/j.tim.2008. 08.006

Ryndak, M. B., Singh, K. K., Peng, Z., Zolla-Pazner, S., Li, H., Meng, L., et al. (2014). Transcriptional profiling of Mycobacterium tuberculosis replicating ex vivo in blood from HIV- and HIV+ subjects. PLoS One 9:e94939. doi: 10.1371/journal. pone.0094939

Saini, D. K., Pant, N., Das, T. K., and Tyagi, J. S. (2002). Cloning, overexpression, purification, and matrix-assisted refolding of devs (Rv 3132c) histidine protein kinase of Mycobacterium tuberculosis. Protein Expr. Purif. 25, 203-208. doi: 10.1006/prep.2002.1628

Sankhe, G. D., Dixit, N. M., and Saini, D. K. (2018). Activation of bacterial histidine kinases: insights into the kinetics of the cis autophosphorylation mechanism. mSphere 3:e00111-18. doi: 10.1128/msphere.00111-118

Schreuder, L. J., Carroll, P., Muwanguzi-Karugaba, J., Kokoczka, R., Brown, A. C., and Parish, T. (2015). Mycobacterium tuberculosis H37Rv has a single nucleotide polymorphism in PHoR which affects cell wall hydrophobicity and gene expression. Microbiology (United Kingdom) 161, 765-773. doi: 10.1099/ mic. 0.000036

Shet, A., Nagaraja, P., and Dixit, N. M. (2016). Viral decay dynamics and mathematical modeling of treatment response: evidence of lower in vivo fitness of HIV-1 Subtype C. J. Acquir. Immune Defic. Syndr. 73, 245-251. doi: 10.1097/ qai.0000000000001101

Soto, C. Y., Menéndez, M. C., Pérez, E., Samper, S., Gómez, A. B., García, M. J., et al. (2004). IS6110 mediates increased transcription of the phoP virulence gene in a multidrug-resistant clinical isolate responsible for tuberculosis outbreaks. J. Clin. Microbiol. 42, 212-219. doi: 10.1128/JCM.42.1.212-219.2004

Stock, A. M., Robinson, V. L., and Goudreau, P. N. (2000). Two-Component signal transduction. Annu. Rev. Biochem. 69, 183-215. doi: 10.1146/annurev.biochem. 69.1 .183

Tekwu, E. M., Sidze, L. K., Assam, J. P. A., Tedom, J. C., Tchatchouang, S., Makafe, G. G., et al. (2014). Sequence analysis for detection of drug resistance in Mycobacterium tuberculosis complex isolates from the central region of cameroon. BMC Microbiol. 14:113. doi: 10.1186/1471-2180-14-113

Vashist, A., Malhotra, V., Sharma, G., Tyagi, J. S., and Clark-Curtiss, J. E. (2018). Interplay of PhoP and DevR response regulators defines expression of the dormancy regulon in virulent Mycobacterium tuberculosis. J. Biol. Chem. 293, 16413-16425. doi: 10.1074/jbc.RA118.004331

Voskuil, M. I., Bartek, I. L., Visconti, K., and Schoolnik, G. K. (2011). The response of Mycobacterium tuberculosis to reactive oxygen and nitrogen species. Front. Microbiol. 2:105. doi: 10.3389/fmicb.2011.00105

Walters, S. B., Dubnau, E., Kolesnikova, I., Laval, F., Daffe, M., and Smith, I. (2006). The Mycobacterium tuberculosis PhoPR two-component system regulates genes essential for virulence and complex lipid biosynthesis. Mol. Microbiol. 60, 312-330. doi: 10.1111/j.1365-2958.2006.05102.x

Wang, S., Engohang-ndong, J., and Smith, I. (2007). Structure of the DNA-Binding domain of the response regulator PhoP from. Biochemistry 46, 14751-14761. doi: 10.1021/bi700970a

Xing, D., Ryndak, M. B., Wang, L., Kolesnikova, I., Smith, I., and Wang, S. (2017). Asymmetric structure of the dimerization domain of PhoR, a sensor kinase important for the virulence of Mycobacterium tuberculosis. ACS Omega 2, 3509-3517. doi: 10.1021/acsomega.7b00612

Zheng, H., Lu, L., Wang, B., Pu, S., Zhang, X., Zhu, G., et al. (2008). Genetic basis of virulence attenuation revealed by comparative genomic analysis of Mycobacterium tuberculosis strain H37Ra versus H37Rv. PLoS One 3:e2375. doi: 10.1371/journal.pone.0002375

Conflict of Interest: The authors declare that the research was conducted in the absence of any commercial or financial relationships that could be construed as a potential conflict of interest.

Publisher's Note: All claims expressed in this article are solely those of the authors and do not necessarily represent those of their affiliated organizations, or those of the publisher, the editors and the reviewers. Any product that may be evaluated in this article, or claim that may be made by its manufacturer, is not guaranteed or endorsed by the publisher.

Copyright (c) 2021 Waturuocha, Krishna, Malhotra, Dixit and Saini. This is an open-access article distributed under the terms of the Creative Commons Attribution License (CC BY). The use, distribution or reproduction in other forums is permitted, provided the original author(s) and the copyright owner(s) are credited and that the original publication in this journal is cited, in accordance with accepted academic practice. No use, distribution or reproduction is permitted which does not comply with these terms. 